\title{
Capacity-Achieving Input Distribution in Per-Sample Zero-Dispersion Model of Optical Fiber
}

\author{
Jihad Fahs, Aslan Tchamkerten and Mansoor I. Yousefi
}

\begin{abstract}
The per-sample zero-dispersion channel model of the optical fiber is considered. It is shown that capacity is uniquely achieved by an input probability distribution that has continuous uniform phase and discrete amplitude that takes on finitely many values. This result holds when the channel is subject to general input cost constraints, that include a peak amplitude constraint and a joint average and peak amplitude constraint.
\end{abstract}

Index terms: Capacity-achieving distributions, optical fiber, zero dispersion.

\section{INTRODUCTION}

Signal propagation in optical fibers can be modeled by the stochastic nonlinear Schrödinger (NLS) equation [1], capturing chromatic dispersion, Kerr nonlinearity, and amplified spontaneous emission (ASE) noise. Finding the capacity and the spectral efficiency of such a channel remains a formidable challenge, even in the special case of zero dispersion. The chief reason for this is that the channel is nonlinear. To date the only non-asymptotic (in input power) capacity result states that the capacity of the optical fiber is upper bounded by $\log (1+\mathrm{SNR})$, where SNR is the signal-to-noise ratio [1], [2].

In this paper we consider the per-sample zero-dispersion (PZD) channel model of the optical fiber. This channel is obtained by setting the dispersion coefficient to zero in the NLS equation and by sampling the output signal at the input signal bandwidth rate $[3$, Sec. III]. These simplifications yield a discrete memoryless channel that maps a complex input $x \in \mathbb{C}$ to a complex output $y \in \mathbb{C}$ through a conditional probability density function (pdf) $p_{Y \mid X}(y \mid x)$. For this channel we show that capacity is uniquely achieved by an input signal that has continuous uniform phase and discrete amplitude that takes on finitely many values independently of the phase. This result holds whenever the input is subject to a peak amplitude constraint, a joint peak amplitude and average cost constraint, or an average cost constraint with a cost function satisfying certain regularity conditions. This proves a conjecture made in [3] and shows that multi-ring modulation formats that are popular in optical fiber communication [4] are indeed optimal for the simplified PZD channel .

\section{Related work}

The conditional pdf in the PZD model can be expressed as an infinite series [3], [5], [6]. The asymptotic capacity of the PZD model is $\frac{1}{2} \log \mathcal{P}+o(1)$ as the average input power $\mathcal{P} \rightarrow \infty$ [3], [6]. Moreover, this asymptotic capacity is achieved by continuous pdfs, up to the $o(1)$ term [3], [5], [6].

Since the work of Smith [7], several authors have established the discreteness of the capacity-achieving input distributions for a variety of channels and input constraints [7]-[17]. Examples include complex additive white Gaussian noise channel [9], linear channels with additive noise [10], [12], [16], [17], and conditionally Gaussian channels [11], [13]-[15].

\section{Contributions}

We first show that the capacity of the PZD channel is uniquely achieved and that the optimal input has a uniform phase that is independent of the amplitude. The main proof ingredient here is a symmetry argument. In a second part we show that the optimal amplitude takes on a finite number of values, following the methodology established by Smith [7]. Although the proof roadmap here is known, implementing it is far from straightforward; unlike channels considered so far where this proof technique is used (see, e.g., [7], [9]-[17]) the PZD channel is non-additive-since the phase noise is a complex function of the input amplitude - and non-conditionally Gaussian.

The paper is organized as follows. In Section [I] we recall the PZD channel model. In Section [II] we present the main result and prove it in Section IV Finally, in Section $\square$ we draw a few concluding remarks.

Jihad Fahs is with the School of Engineering, Lebanese American University, Beirut 1102 2801, Lebanon (e-mail: jihad.fahs@lau.edu.lb). Aslan Tchamkerten and Mansoor I. Yousefi are with the Communications and Electronics Department, Télécom ParisTech, Université Paris-Saclay, 75013 Paris, France (e-mails: \{aslan.tchamkerten,yousefi\}@telecom-paristech.fr). 


\section{Notation}

Random variables and their realizations are denoted by upper and lower case letters, respectively. Real, non-negative real, integer, positive integer, non-negative integer, and complex numbers are respectively denoted by $\mathbb{R}, \mathbb{R} 0_{0}^{+}, \mathbb{Z}, \mathbb{N}, \mathbb{N}$ and $\mathbb{C}$. The real and imaginary parts of $x \in \mathbb{C}$ are denoted by $\Re(x)$ and $\Im(x)$, respectively. The cumulative distribution function (cdf) and the pdf of a random variable $X$ are denoted by $F_{X}(x)$ and $p_{X}(x)$, respectively. The expected value of a random variable $X$ is $\mathrm{E}(X)$. The uniform distribution on the interval $[a, b)$ is denoted by $\mathcal{U}(a, b)$. Given two functions $f(x): \mathbb{R} \mapsto \mathbb{R}$ and $g(x): \mathbb{R} \mapsto \mathbb{R}$ we use the following standard order notations. We write $f(x)=\omega(g(x))$, or equivalently $g(x)=o(f(x))$, if for any $k>0$ there exists a $c>0$ such that $|f(x)|>k|g(x)|$ for all $|x| \geq c$. We write $f(x)=\Omega(g(x))$ if there exists a $k>0$ and $c>0$ such that $|f(x)|>k|g(x)|$ for all $|x| \geq c$. Finally, we write $f(x) \equiv g(x)$ as $x \rightarrow x_{0}$ if $\lim _{x \rightarrow x_{0}} \frac{f(x)}{g(x)}=1$.

\section{The PER-SAMPle ZERo-DisPersion CHANNEL}

We briefly recall the PZD channel model derived in [3, Sec. III. A, Eq. 18]. The reader familiar with this model may move on to the conditional pdf (3).

Let $Q(t, z)$ denote the complex envelope of the signal as a function of time $t$ and distance $z$ along the fiber. The propagation of the signal in the optical fiber is described by a partial differential equation known as the stochastic NLS equation [4, Chap. 2,3]. Setting the dispersion to zero in equation [1, Eq. 7, with $\beta_{2}=0$ ], we obtain the following stochastic ordinary differential equation

$$
\frac{d Q(t, z)}{d z}=j \gamma Q(t, z)|Q(t, z)|^{2}+N(t, z), \quad 0 \leq z \leq \mathcal{L}
$$

where $\gamma$ is the nonlinearity coefficient, $\mathcal{L}$ is the fiber length, and $j \stackrel{\text { def }}{=} \sqrt{-1}$. Furthermore, $N(t, z)$ is (zero-mean) circularly symmetric complex Gaussian noise satisfying

$$
\mathrm{E}\left(N(t, z) N^{*}\left(t^{\prime}, z^{\prime}\right)\right)=\sigma_{0}^{2} \delta_{\mathcal{W}}\left(t-t^{\prime}\right) \delta\left(z-z^{\prime}\right)
$$

where $\sigma_{0}^{2}$ is the noise in-band power spectral density, $\delta(x)$ is the Dirac delta function, and $\delta_{\mathcal{W}}(x) \stackrel{\text { def }}{=} \mathcal{W}$ sinc $(\mathcal{W} x)$, in which $\operatorname{sinc}(x) \stackrel{\text { def }}{=} \sin (\pi x) /(\pi x)$, and $\mathcal{W}$ is the noise bandwidth.

Equation (1) defines a continuous-time channel from the input $Q(t, 0)$ to the output $Q(t, \mathcal{L})$. To obtain an equivalent discretetime model we need to sample $Q(t, 0)$ at rate $\mathcal{W}(0)$ and $Q(t, \mathcal{L})$ at rate $\mathcal{W}(\mathcal{L})$, where $\mathcal{W}(z)$ denotes the signal bandwidth at distance $z$. Because of the nonlinearity, the signal bandwidth changes along the fiber and therefore in general $\mathcal{W}(\mathcal{L}) \neq \mathcal{W}(0)$ [3, Sec. VIII], [18]. How the bandwidth changes as a function of distance remains an important open problem in optical fiber communication. The PZD channel arises by assuming a sub-optimal receiver that samples the output signal at the input rate $1 / \mathcal{W}, \mathcal{W}=\mathcal{W}(0)$. This channel is discrete-time, memoryless, and stationary and maps, at rate $1 / \mathcal{W}$, an input $X \in \mathbb{C}$ to a random output $Y \in \mathbb{C}$. The input output relation is obtained by solving (1) [3, Eq. 30]

$$
Y=[X+W(\mathcal{L})] e^{j \gamma \int_{0}^{\mathcal{L}}|X+W(\ell)|^{2} d \ell},
$$

where $W(\ell)$ is a complex Wiener process with variance $\ell \sigma^{2}, \sigma^{2}=\sigma_{0}^{2} \mathcal{W}$. Letting $\left(R_{0}, \Phi_{0}\right)$ and $(R, \Phi)$ denote the polar coordinates of $X$ and $Y$, respectively, equation (2) equivalently defines the conditional pdf (see [5], [6], [3, Eq. 18])

$$
p_{R, \Phi \mid R_{0}, \Phi_{0}}\left(r, \phi \mid r_{0}, \phi_{0}\right) \stackrel{\text { def }}{=} p\left(r, \phi \mid r_{0}, \phi_{0}\right)=\frac{1}{2 \pi} p_{R \mid R_{0}}\left(r \mid r_{0}\right)+\frac{1}{\pi} \sum_{m=1}^{+\infty} \Re\left(C_{m}\left(r, r_{0}\right) e^{j m\left(\phi-\phi_{0}-\gamma r_{0}^{2} \mathcal{L}\right)}\right)
$$

where

$$
\begin{gathered}
p_{R \mid R_{0}}\left(r \mid r_{0}\right) \stackrel{\text { def }}{=} \frac{2 r}{\sigma^{2} \mathcal{L}} e^{-\frac{r^{2}+r_{0}^{2}}{\sigma^{2} \mathcal{L}}} I_{0}\left(\frac{2 r r_{0}}{\sigma^{2} \mathcal{L}}\right), \\
C_{m}\left(r, r_{0}\right) \stackrel{\text { def }}{=} r b_{m} e^{-a_{m}\left(r^{2}+r_{0}^{2}\right)} I_{m}\left(2 b_{m} r_{0} r\right),
\end{gathered}
$$

where $I_{m}(\cdot)$ is the modified Bessel function of the first kind and where

$$
\begin{aligned}
& a_{m} \stackrel{\text { def }}{=} \frac{\sqrt{j m \gamma}}{\sigma} \operatorname{coth}\left(\sqrt{j m \gamma \sigma^{2}} \mathcal{L}\right), \\
& b_{m} \stackrel{\text { def }}{=} \frac{\sqrt{j m \gamma}}{\sigma} \frac{1}{\sinh \left(\sqrt{j m \gamma \sigma^{2}} \mathcal{L}\right)} .
\end{aligned}
$$

Note that, because of the potentially sub-optimal discretization of the output, the capacity of the PZD channel (3) (measured in bits/s) is at most equal to the capacity of the continuous-time zero-dispersion channel (1) [3]. 


\section{MAIN RESULT}

We consider the PZD channel (3) when the input $\left(R_{0}, \Phi_{0}\right)$ is subject to one of the following constraints.

Peak amplitude constraint: The cdf of $R_{0}$ belongs to the set

$$
\mathcal{P} \stackrel{\text { def }}{=}\left\{F_{R_{0}}\left(r_{0}\right): \int_{0}^{\rho} d F_{R_{0}}\left(r_{0}\right)=1\right\},
$$

for some $0<\rho<\infty$.

Average cost constraint. The cdf of $R_{0}$ belongs to the set

$$
\mathcal{A} \stackrel{\text { def }}{=}\left\{F_{R_{0}}\left(r_{0}\right): \int_{0}^{+\infty} \mathcal{C}\left(r_{0}\right) d F_{R_{0}}\left(r_{0}\right) \leq A\right\}
$$

for some $0<A<\infty$ and cost function $\mathcal{C}\left(r_{0}\right): \mathbb{R}_{0}^{+} \mapsto \mathbb{R}_{0}^{+}$that satisfies the following conditions:

C1. $\mathcal{C}\left(r_{0}\right)$ is lower semi-continuous, non-decreasing, $\mathcal{C}(0)=0$ and $\lim _{r_{0} \rightarrow+\infty} \mathcal{C}\left(r_{0}\right)=+\infty$;

C2. $\mathcal{C}\left(r_{0}\right)$ can be analytically extended from $[0, \infty)$ to a horizontal strip in the complex plane

$$
\mathcal{S}_{\delta}=\{z \in \mathbb{C}:|\Im(z)|<\delta,, \delta>0\},
$$

for some $\delta>0$;

C3. $\mathcal{C}\left(r_{0}\right)=\omega\left(r_{0}^{2}\right)$.

Joint peak amplitude and average cost constraint. The cdf of $R_{0}$ belongs to the set $\mathcal{P} \cap \mathcal{A}^{\prime}$, where $\mathcal{A}^{\prime}$ is defined as $\mathcal{A}$ but with condition $\mathrm{C} 3$ replaced by the weaker condition $\mathrm{C} 3: \mathcal{C}\left(r_{0}\right)=\omega\left(\ln r_{0}\right)$.

An example of a family of cost functions satisfying conditions C1-C2 is $\mathcal{C}\left(r_{0}\right)=r_{0}^{q}$ for $q \in \mathbb{N}$.

Abusing slightly notation, we will use $X$ and $Y$ to denote both the complex values and their representations in polar coordinates. With this convention, the channel capacity is

$$
C=\sup _{F_{X}: F_{R_{0}}\left(r_{0}\right) \in \mathcal{F}} I(X ; Y),
$$

where $I(X ; Y)$ denotes the mutual information and $\mathcal{F}$ represents any of the sets $\mathcal{P}, \mathcal{A}$, or $\mathcal{P} \cap \mathcal{A}^{\prime}$.

Theorem 1 The channel capacity in (10) is finite and is achieved by a unique cdf $F_{R_{0}^{*}, \Phi_{0}^{*}}\left(r_{0}, \phi_{0}\right)$ where the phase $\Phi_{0}^{*}$ is uniform over $[0,2 \pi)$ and where the amplitude $R_{0}^{*}$ takes on a finite number of values independently of $\Phi_{0}^{*}$.

Hence, under fairly general conditions the support of the optimal input consists of a finite number of concentric rings in the complex plane. Without a peak constraint, the same result holds provided that the cost function grows faster than $r_{0}^{2}$. Whether the conclusion extends to a strict average power constraint, that is with cost function $\mathcal{C}\left(r_{0}\right)=r_{0}^{2}$, remains an open problem.

Remark 1 Theorem 11 still holds when the input is subject to a finite number of constraints given by cost functions $\mathcal{C}_{i}\left(r_{0}\right)$, $1 \leq i \leq M<\infty$, satisfying C1-C2, and such that $\mathcal{C}_{i}\left(r_{0}\right)=\omega\left(r_{0}^{2}\right)$ for at least one cost constraint. For example, we may consider a joint second and a fourth moment constraint as in the non-coherent Rician fading channel [14].

\section{Proof of Theorem 1}

To prove Theorem 1 we first show that capacity is finite and achieved by a unique cdf. Then, we show that the optimal input phase is uniform and independent of the optimal input amplitude. Finally, we prove that the optimal input amplitude takes on a finite number of values.

Lemma 1 The capacity (10) is finite and achieved by a unique cdf.

Proof: It is known that that the set of input distributions satisfying an input constraint in Section [II] is compact [19, Thm. 3], [7, Prop. 1]. Further, in Appendix 【 we prove that mutual information $I(X ; Y)$ is continuous in $F_{X}$. Therefore, from the extreme value theorem [20, Thm. 4.16], the supremum in (10) is finite and achieved.

For uniqueness, it suffices to prove that $I(X ; Y)=h(Y)-h(Y \mid X)$ is a strictly concave function of $F_{X}$. The term $h(Y \mid X)$ as a function of $F_{X}$ is linear hence concave. The term $h(Y)$ is strictly concave in $F_{Y}$. Below, we prove that the linear mapping $F_{X} \mapsto F_{Y}$ is injective which then implies that $h(Y)$, and therefore $I(X ; Y)$, is strictly concave in $F_{X}$.

Changing variable $z^{\prime}=\mathcal{L}-z$ in (1), $X$ is obtained from $Y$ according to (1) with $\gamma \mapsto-\gamma$ and $N(t, z) \mapsto-N\left(t, \mathcal{L}-z^{\prime}\right)$. Since $-N$ and $N$ are identically distributed, we obtain the symmetry relation $p_{X \mid Y}(x \mid y ; \gamma)=p_{Y \mid X}(x \mid y ;-\gamma)$. The channel is therefore invertible and injective. 


\section{A. Optimal input phase}

In this section we show that the optimal input phase is uniform, independently of the optimal input amplitude.

Lemma 2 The following properties hold:

1) the output amplitude $R$ is independent of the input phase $\Phi_{0}$;

2) the capacity-achieving input in (10) has uniform phase $\Phi_{0}^{*} \sim \mathcal{U}(0,2 \pi)$ independent of the amplitude $R_{0}^{*}$, i.e.,

$$
d F_{\left(R_{0}^{*}, \Phi_{0}^{*}\right)}\left(r_{0}, \phi_{0}\right)=\frac{1}{2 \pi} d \phi_{0} d F_{R_{0}^{*}}\left(r_{0}\right) .
$$

Proof: 1) From (2)

$$
\begin{aligned}
R & =\left|R_{0} e^{j \Phi_{0}}+W(\mathcal{L})\right| \\
& =\left|R_{0}+W^{\prime}(\mathcal{L})\right| \\
& \stackrel{d}{=}\left|R_{0}+W(\mathcal{L})\right|,
\end{aligned}
$$

where $W^{\prime}(\mathcal{L}) \stackrel{\text { def }}{=} e^{-j \Phi_{0}} W(\mathcal{L})$. The last equality is in distribution where we used the fact that $W^{\prime}(\mathcal{L})$ and $W(\mathcal{L})$ are identically distributed from the circularly symmetry property.

2) From (2) and by the circularly symmetry of the complex Wiener process, if output $Y$ corresponds to input $X$ then output $e^{j \theta} Y$ corresponds to input $e^{j \theta} X$, for any fixed $\theta \in[0,2 \pi)$. Therefore, the mutual information is invariant under an input rotation. Hence, if $X^{*}$ is capacity-achieving, so is $e^{j \theta} X^{*}$ for any $\theta \in[0,2 \pi)$. By Lemma 1 , the capacity-achieving input distribution is unique. This implies that $X^{*}$ has a uniform phase $\Phi_{0}^{*} \sim \mathcal{U}(0,2 \pi)$, that is independent of the amplitude.

Lemmas 1 and 2 yield:

Proposition 1 The capacity (10) simplifies to

$$
C=\max _{F_{R_{0}} \in \mathcal{F}} I\left(F_{R_{0}}\right),
$$

where

$$
\begin{aligned}
I\left(F_{R_{0}}\right) & \stackrel{\text { def }}{=} I\left(R_{0}, \Phi_{0}^{*} ; R, \Phi\right) \\
& =h(R)+\ln (2 \pi)-h\left(R, \Phi \mid R_{0}, \Phi_{0}^{*}\right),
\end{aligned}
$$

with $\Phi_{0}^{*} \sim \mathcal{U}(0,2 \pi)$.

From Proposition 1, finding capacity reduces to maximizing the strictly concave function $I\left(F_{R_{0}}\right)$. The set $\mathcal{F}$ is linear, and thus weakly differentiable, in $F_{R_{0}}$. Extending a result in [17] for additive noise channels to the PZD channel, it can be shown that $I\left(F_{R_{0}}\right)$ is weakly differentiable. As a result, the KKT conditions characterize the optimal input $F_{R_{0}^{*}}$ in terms of necessary and sufficient conditions.

\section{B. KKT conditions.}

The derivation of the KKT conditions can be found in [7, Corollary 1] for the peak amplitude constraint, and in [15, Theorem 15] for the average cost constraint. We present their final forms below.

Peak amplitude constraint. An input amplitude $R_{0}^{*}$ with $\operatorname{cdf} F_{0}^{*} \in \mathcal{P}$ achieves the capacity $C$ in (11) if and only if

$$
\begin{aligned}
\operatorname{LHS}_{\rho}\left(r_{0}\right) & \stackrel{\text { def }}{=} C-\ln (2 \pi)+\int_{0}^{+\infty} p\left(r \mid r_{0}\right) \ln p\left(r ; F_{0}^{*}\right) d r+\frac{1}{2 \pi} \int_{0}^{2 \pi} h\left(R, \Phi \mid r_{0}, \phi_{0}\right) d \phi_{0} \\
& \geq 0,
\end{aligned}
$$

for all $0 \leq r_{0} \leq \rho$, with equality if $r_{0}$ is a point of increase of $F_{0}^{*}$.

Average cost constraint. An input amplitude $R_{0}^{*}$ with $\operatorname{cdf} F_{0}^{*} \in \mathcal{A}$ achieves the capacity $C$ in (11) if and only if there exists $\nu \geq 0$ such that

$$
\begin{aligned}
\operatorname{LHS}_{A}\left(r_{0}\right) & \stackrel{\text { def }}{=} C-\ln (2 \pi)+\int_{0}^{+\infty} p\left(r \mid r_{0}\right) \ln p\left(r ; F_{0}^{*}\right) d r+\nu\left(\mathcal{C}\left(r_{0}\right)-A\right)+\frac{1}{2 \pi} \int_{0}^{2 \pi} h\left(R, \Phi \mid r_{0}, \phi_{0}\right) d \phi_{0} \\
& \geq 0,
\end{aligned}
$$

for all $r_{0} \geq 0$, with equality if $r_{0}$ is a point of increase of $F_{0}^{*}$.

Joint peak amplitude and average cost constraint. The KKT condition in this case is same as (13), but for all $0 \leq r_{0} \leq \rho$. 


\section{Discreteness of optimal input}

Following Smith [7], we apply the identity theorem [21, Thm. 10.26] to the KKT conditions. The first part of the argument is to show that $\operatorname{LHS}_{\rho}\left(r_{0}\right)$ and $\operatorname{LHS}_{A}\left(r_{0}\right)$ in (12)-(13) can be analytically extended from $r_{0} \in \mathbb{R}_{0}^{+}$to an open connected region $\mathcal{O}_{\delta}, \delta>0$, in the complex plane. This is established in Lemma 9 in Appendix IT The second part of the argument consists in deriving the optimality of discrete inputs by way of contradiction in the identity theorem.

Peak amplitude constraint. Suppose that the points of increase of $F_{0}^{*}$ have an accumulation point in the interval $[0, \rho]$. Then, since $\operatorname{LHS}_{\rho}(z)$ is analytic on $\mathcal{O}_{\delta}$, it is identically zero on $\mathcal{O}_{\delta}$ from the identity theorem [21, Thm. 10.26]. Therefore the KKT condition (12) is satisfied with equality for all $r_{0} \geq 0$.

Using the upper bound on $\operatorname{LHS}_{\rho}\left(r_{0}\right)$ in Lemma 10 in Appendix [II] there exists $K>0$ such that

$$
\begin{aligned}
0 & =\operatorname{LHS}_{\rho}\left(r_{0}\right) \\
& \leq C+\ln \left(\frac{1}{K}\right)+\frac{r_{0}^{2}}{\sigma^{2} \mathcal{L}}-\ln \left(1-\xi\left(r_{0}\right)\right)+\left(\rho-(1-\epsilon) r_{0}\right) \sqrt{\frac{\pi}{\sigma^{2} \mathcal{L}}} \mathrm{L}_{\frac{1}{2}}\left(-\frac{r_{0}^{2}}{\sigma^{2} \mathcal{L}}\right),
\end{aligned}
$$

where $\mathrm{L}_{\frac{1}{2}}(\cdot)$ is a Laguerre polynomial, $\xi\left(r_{0}\right) \rightarrow 0$ as $r \rightarrow+\infty$, and $\epsilon \in(0,1)$. Since $\mathrm{L}_{\lambda}(x) \equiv \frac{|x|^{\lambda}}{\Gamma(1+\lambda)}$ as $x \rightarrow-\infty$ [22], we have

$$
\lim _{r_{0} \rightarrow+\infty} \frac{r_{0} \sqrt{\frac{\pi}{\sigma^{2} \mathcal{L}}} \mathrm{L}_{\frac{1}{2}}\left(-\frac{r_{0}^{2}}{\sigma^{2} \mathcal{L}}\right)}{r_{0}^{2}}=\frac{2}{\sigma^{2} \mathcal{L}} .
$$

Dividing (14) by $r_{0}^{2}>0$ and taking the limit as $r_{0} \rightarrow+\infty$, we obtain

$$
0 \leq \frac{1-2(1-\epsilon)}{\sigma^{2} \mathcal{L}}, \quad \forall \epsilon \in(0,1),
$$

where we used the fact that $\lim _{r_{0} \rightarrow+\infty} \xi\left(r_{0}\right)=0$ (see Lemma 4). Since (16) holds for any $0<\epsilon<1$, choosing $\epsilon<\frac{1}{2}$ yields a contradiction.

It follows that the points of increase of $F_{0}^{*}$ are isolated. Since the interval $[0, \rho]$ is bounded, there are a finite number of mass points.

Average cost constraint. Consider the analytic extension $\operatorname{LHS}_{A}(z)$ of $\operatorname{LHS}_{A}\left(r_{0}\right)$ in $\mathcal{O}_{\delta}$ and suppose that $F_{0}^{*}$ has an infinite number of points of increase in a bounded interval in $\mathbb{R}_{0}^{+}$. Then, from the identity theorem, $\operatorname{LHS}_{A}(z)$ is identically zero on $\mathcal{O}_{\delta}$. As a result, the KKT condition (13) is satisfied with equality for all $r_{0} \geq 0$ and we have

$$
\begin{aligned}
0 & =\operatorname{LHS}_{A}\left(r_{0}\right) \\
& >\nu\left(\mathcal{C}\left(r_{0}\right)-A\right)+C+\ln \left(\frac{k_{1}}{2 \pi k_{u}}\right)+\frac{1}{\sigma^{2} \mathcal{L}} r_{0}^{2}-r_{0} \sqrt{\frac{\pi}{\sigma^{2} \mathcal{L}}} \mathrm{L}_{\frac{1}{2}}\left(-\frac{r_{0}^{2}}{\sigma^{2} \mathcal{L}}\right),
\end{aligned}
$$

where (17) follows from the lower bound on $\operatorname{LHS}_{A}\left(r_{0}\right)$ in Lemma 11 in Appendix [II] Dividing [17) by $r_{0}^{2}>0$ and taking the limit $r_{0} \rightarrow+\infty$ gives

$$
\nu \lim _{r_{0} \rightarrow+\infty} \frac{\mathcal{C}\left(r_{0}\right)}{r_{0}^{2}}-\frac{1}{\sigma^{2} \mathcal{L}}<0
$$

where we used (15). The inequality (18) is impossible for $\mathcal{C}\left(r_{0}\right)=\omega\left(r_{0}^{2}\right)$, unless $\nu=0$ which is ruled out in [17, Lemma 5].

We now prove that the number of mass points is finite. Suppose that $F_{0}^{*}$ has an infinite number of points of increase with only a finite number of them in any bounded interval in $\mathbb{R}_{0}^{+}$. Then, the points of increase tend to infinity. We establish in Lemma 11 in Appendix [II] a lower bound on $\operatorname{LHS}_{A}\left(r_{0}\right)$ which diverges to $+\infty$ as $r_{0} \rightarrow+\infty$. This implies that LHS $_{A}\left(r_{0}\right) \neq 0$ at large values of $r_{0}$ which contradicts the possibility of having arbitrarily large mass points. It follows that $F_{0}^{*}$ has a finite number of isolated points of increase in $\mathbb{R}_{0}^{+}$.

The above proof immediately generalize to the case with multiple average cost constraints, described in Remark 1 .

Joint average cost and peak amplitude constraints. Suppose that $F_{0}^{*}$ has an infinite number of points of increase in $[0, \rho]$. Then the KKT condition (13) implies that

$$
\operatorname{LHS}_{A}\left(r_{0}\right)=0, \quad \forall r_{0} \geq 0 .
$$

Thus (18) holds true and

$$
\nu \lim _{r_{0} \rightarrow+\infty} \frac{\mathcal{C}\left(r_{0}\right)}{r_{0}^{2}}<\frac{1}{\sigma^{2} \mathcal{L}}
$$

On the other hand, since the support of $F_{0}^{*}$ is restricted to $[0, \rho]$, we can apply the upper bound in Lemma 10 to the KKT condition (13) to obtain

$$
\nu\left(\mathcal{C}\left(r_{0}\right)-A\right)+C+\ln \left(\frac{1}{K}\right)+\frac{r_{0}^{2}}{\sigma^{2} \mathcal{L}}-\ln \left(1-\xi\left(r_{0}\right)\right)+\left(\rho-(1-\epsilon) r_{0}\right) \sqrt{\frac{\pi}{\sigma^{2} \mathcal{L}}} \mathrm{L}_{\frac{1}{2}}\left(-\frac{r_{0}^{2}}{\sigma^{2} \mathcal{L}}\right) \geq 0 .
$$


Dividing by $r_{0}^{2}>0$ and letting $r_{0} \rightarrow+\infty$ gives

$$
\nu \lim _{r_{0} \rightarrow+\infty} \frac{\mathcal{C}\left(r_{0}\right)}{r_{0}^{2}} \geq \frac{2(1-\epsilon)-1}{\sigma^{2} \mathcal{L}}, \quad \forall \epsilon \in(0,1) .
$$

Taking the limit $\epsilon \rightarrow 0$

$$
\nu \lim _{r_{0} \rightarrow+\infty} \frac{\mathcal{C}\left(r_{0}\right)}{r_{0}^{2}} \geq \frac{1}{\sigma^{2} \mathcal{L}}
$$

Equations (20) and (21) establish a contradiction. It follows that $F_{0}^{*}$ has a finite number of isolated points of increase in $[0, \rho]$.

\section{CONCLUSIONS}

We studied the capacity-achieving input distribution in the per-sample zero-dispersion model of the optical fiber, subject to a peak amplitude, an average cost, or a joint cost constraint. We proved that the capacity-achieving input distribution in this channel is unique, has a uniform phase that is independent of the amplitude, and the distribution of the amplitude is discrete with a finite number of mass points. In other words, multi-ring modulation formats commonly used in optical communication can achieve channel capacity_potentially with non-uniform ring spacing and probabilities. Whether such constellations are optimal for the dispersive optical fiber is an interesting open problem.

\section{APPENDIX I \\ CONTINUITY OF THE MUTUAL INFORMATION}

In this appendix we prove that $I(X ; Y)$ in the channel (3) is continuous in $F_{X} \in \mathcal{F}$. First, we establish upper and lower bounds on the conditional pdf in Lemma 4, upper and lower bounds on the output pdf in Lemma 5, and an upper bound on the conditional entropy density in Lemma 6 . These bounds are then used to prove the continuity of the conditional entropy in Lemma 7 and the continuity of the output entropy in Lemma 8 Lemmas 7 and 8 yield the desired result.

Note that, since the function $i(p)=-p \ln (p)$ has opposite signs when $0<p<1$ and $p>1$, to upper-bound entropies, we need both upper and lower bounds on the probability distributions.

We begin by recalling a few properties of the modified Bessel function.

Lemma 3 (Bounds on the Bessel Functions) The modified Bessel functions of the first kind $I_{m}(x)$ satisfy the following properties:

1) $I_{0}(x) \geq 1$ when $x \geq 0$, with equality iff $x=0$. Furthermore, $I_{m}(x) \geq 0$ when $x \geq 0, m \in \mathbb{N}_{0}$;

2) $\left|I_{m}(z)\right| \leq\left|I_{0}(\Re(z))\right|$ when $z \in \mathbb{C}, m \in \mathbb{N}_{0}$. Furthermore, $I_{m}(x) \leq e^{|x|}$ when $x \in \mathbb{R}, m \in \mathbb{N}_{0}$;

3) If $m \in \mathbb{N}_{0}$ and $z \rightarrow 0$

$$
I_{m}(z) \equiv \frac{\left(\frac{1}{2} z\right)^{m}}{m !}
$$

4) For any $0<\epsilon<1$, there exists a $K>0$ such that $I_{0}(x) \geq K e^{(1-\epsilon) x}$, when $x \geq 0$;

5) $I_{m}(x)$ is monotonically increasing for $x \geq 0$ and $m \in \mathbb{N}_{0}$;

6) $I_{m}(z)$ is an analytic function of $z$ in the entire complex plane for $m \in \mathbb{Z}$.

Proof: The proof of the well-known inequalities in 1) and 2) is straightforward using the integral definition of $I_{m}(x)$, $m \in \mathbb{N}_{0}$ [22, Prop. 9.6.19]. Property 3) can be found in [22, Prop. 9.6.7]. For the inequality in 4), note that $e^{(\epsilon-1) x} I_{0}(x) \equiv \frac{e^{\epsilon x}}{\sqrt{2 \pi x}}$ as $x \rightarrow+\infty$ [22, Prop. 9.7.1]. Therefore the positive and continuous function $e^{(\epsilon-1) x} I_{0}(x)$ is lower bounded by some $K>0$. The proof of 5) is due to 1) in this Lemma, and because $d I_{m}(x) / d x=I_{m+1}(x)+\frac{m}{x} I_{m}(x)$ [22, Prop. 9.6.26]. Property 6) can be found in [22, Prop. 9.6.1].

Lemma 4 (Bounds on the Conditional pdf) The conditional pdf (3) satisfies the following inequalities.

1) Upper bound:

$$
p\left(r, \phi \mid r_{0}, \phi_{0}\right)<k_{u} p_{R \mid R_{0}}\left(r \mid r_{0}\right)
$$

where

$$
k_{u} \stackrel{\text { def }}{=} \frac{1}{2 \pi}\left(1+\sqrt{2} \sum_{m=1}^{+\infty} \frac{\beta_{m}}{\sinh \left(\beta_{m}\right)}\right)<\infty
$$


in which $\beta_{m} \stackrel{\text { def }}{=} \sqrt{\frac{m \gamma}{2}} \sigma \mathcal{L}, m>0$. The conditional pdf of the amplitude is upper bounded as

$$
\begin{aligned}
p_{R \mid R_{0}}\left(r \mid r_{0}\right) & \leq \frac{2 r}{\sigma^{2} \mathcal{L}} e^{-\frac{\left(r-r_{0}\right)^{2}}{\sigma^{2} \mathcal{L}}} \\
& \leq \frac{2 r}{\sigma^{2} \mathcal{L}} .
\end{aligned}
$$

2) Lower bound:

$$
p\left(r, \phi \mid r_{0}, \phi_{0}\right) \geq \frac{1}{2 \pi} p_{R \mid R_{0}}\left(r \mid r_{0}\right)\left(1-\xi\left(r_{0}\right)\right),
$$

where

$$
\xi\left(r_{0}\right)=\sqrt{2} e^{-\left(\Re\left(a_{1}\right)-\frac{1}{\sigma^{2} \mathcal{L}}\right) r_{0}^{2}} \sum_{m=1}^{+\infty} \frac{\beta_{m}}{\sinh \left(\beta_{m}\right)} .
$$

We have $\xi\left(r_{0}\right) \rightarrow 0$ as $r_{0} \rightarrow+\infty$

Proof:

We apply the following inequalities to the conditional pdf (3)

$$
-\left|C_{m}\left(r, r_{0}\right)\right| \leq \Re\left(C_{m}\left(r, r_{0}\right) e^{j m\left(\phi-\phi_{0}-\gamma r_{0}^{2} \mathcal{L}\right)}\right) \leq\left|C_{m}\left(r, r_{0}\right)\right|,
$$

where the expression of $C_{m}\left(r, r_{0}\right)$ is given by equation (5). First, we upper-bound $C_{m}\left(r, r_{0}\right)$ as follows

$$
\begin{aligned}
\left|C_{m}\left(r, r_{0}\right)\right| & =\left|r b_{m} e^{-a_{m}\left(r^{2}+r_{0}^{2}\right)} I_{m}\left(2 b_{m} r_{0} r\right)\right| \\
& \leq r\left|b_{m}\right| e^{-\Re\left(a_{m}\right)\left(r^{2}+r_{0}^{2}\right)} I_{0}\left(2 \Re\left(b_{m}\right) r_{0} r\right) .
\end{aligned}
$$

We upper-bound $\left|b_{m}\right|$ and $\Re\left(b_{m}\right)$, and lower-bound $\Re\left(a_{m}\right)$, in (29).

Using the expression for $a_{m}$ in (6), we have $\Re\left(a_{m}\right) \stackrel{\text { def }}{=} \frac{1}{\sigma^{2} \mathcal{L}} t\left(\beta_{m}\right)$ where $\beta_{m} \stackrel{\text { def }}{=} \sqrt{\frac{m \gamma}{2}} \sigma \mathcal{L}, m>0$, and where

$$
t(x)=\frac{x(\sinh (2 x)+\sin (2 x))}{2\left(\sinh ^{2}(x)+\sin ^{2}(x)\right)} .
$$

It can be verified that $t(x)$ is increasing for $x>0$ and $\lim _{x \rightarrow 0} t(x)=1$. Thus, $t\left(\beta_{m}\right) \geq t\left(\beta_{1}\right)>t\left(\beta_{0}\right)=1$ when $m \geq 1$, and we obtain two lower bounds

$$
\begin{aligned}
\Re\left(a_{m}\right) & \geq \Re\left(a_{1}\right) \\
& >\frac{1}{\sigma^{2} \mathcal{L}} .
\end{aligned}
$$

Similarly, using the expression for $b_{m}$ in (7), we obtain

$$
\begin{aligned}
\Re\left(b_{m}\right) & =\frac{\beta_{m}}{\sigma^{2} \mathcal{L}} \frac{\sinh \left(\beta_{m}\right) \cos \left(\beta_{m}\right)+\cosh \left(\beta_{m}\right) \sin \left(\beta_{m}\right)}{\sinh ^{2}\left(\beta_{m}\right)+\sin ^{2}\left(\beta_{m}\right)} \\
& \leq \frac{1}{\sigma^{2} \mathcal{L}},
\end{aligned}
$$

and

$$
\begin{aligned}
\left|b_{m}\right| & =\frac{\sqrt{2} \beta_{m}}{\sigma^{2} \mathcal{L} \sqrt{\sinh ^{2}\left(\beta_{m}\right)+\sin ^{2}\left(\beta_{m}\right)}} \\
& \leq \frac{\sqrt{2} \beta_{m}}{\sigma^{2} \mathcal{L} \sinh \left(\beta_{m}\right)} .
\end{aligned}
$$

Substituting (31)- (34) into (29), we obtain two upper bounds on $C_{m}\left(r, r_{0}\right)$

$$
\begin{aligned}
\left|C_{m}\left(r, r_{0}\right)\right| & \leq \frac{\sqrt{2} r}{\sigma^{2} \mathcal{L}} \frac{\beta_{m}}{\sinh \left(\beta_{m}\right)} e^{-\Re\left(a_{1}\right)\left(r^{2}+r_{0}^{2}\right)} I_{0}\left(\frac{2 r_{0} r}{\sigma^{2} \mathcal{L}}\right) \\
& <\frac{\sqrt{2} r}{\sigma^{2} \mathcal{L}} \frac{\beta_{m}}{\sinh \left(\beta_{m}\right)} e^{-\frac{r^{2}+r_{0}^{2}}{\sigma^{2} \mathcal{L}}} I_{0}\left(\frac{2 r_{0} r}{\sigma^{2} \mathcal{L}}\right) .
\end{aligned}
$$

Upper Bound. 
Applying the upper bound in (28) to the conditional pdf (3) and using the second upper bound on $C_{m}\left(r, r_{0}\right)$ in (36)

$$
\begin{aligned}
p\left(r, \phi \mid r_{0}, \phi_{0}\right) & \leq \frac{1}{2 \pi} p_{R \mid R_{0}}\left(r \mid r_{0}\right)+\frac{1}{\pi} \sum_{m=1}^{+\infty}\left|C_{m}\left(r, r_{0}\right)\right| \\
& <\frac{1}{2 \pi} p_{R \mid R_{0}}\left(r \mid r_{0}\right)+\frac{1}{\pi} \frac{\sqrt{2} r}{\sigma^{2} \mathcal{L}} e^{-\frac{\left(r^{2}+r_{0}^{2}\right)}{\sigma^{2} \mathcal{L}}} I_{0}\left(\frac{2 r_{0} r}{\sigma^{2} \mathcal{L}}\right) \sum_{m=1}^{+\infty} \frac{\beta_{m}}{\sinh \left(\beta_{m}\right)} \\
& =k_{u} p_{R \mid R_{0}}\left(r \mid r_{0}\right),
\end{aligned}
$$

where $k_{u}$ is defined in 23). It can be verified that $k_{u}<\infty$.

The upper bound on $p_{R \mid R_{0}}\left(r \mid r_{0}\right)$ follows from applying the inequality in Lemma 3 - 2 to the conditional pdf (4).

Lower Bound.

Applying the lower bound in (28) to the conditional pdf (3) and using the first upper bound on $C_{m}\left(r, r_{0}\right)$ in 35

$$
\begin{aligned}
p\left(r, \phi \mid r_{0}, \phi_{0}\right) & \geq \frac{1}{2 \pi} p_{R \mid R_{0}}\left(r \mid r_{0}\right)-\frac{1}{\pi} \sum_{m=1}^{+\infty}\left|C_{m}\left(r, r_{0}\right)\right| \\
& \geq \frac{1}{2 \pi} p_{R \mid R_{0}}\left(r \mid r_{0}\right)-\frac{1}{\pi} \frac{\sqrt{2} r}{\sigma^{2} \mathcal{L}} e^{-\Re\left(a_{1}\right)\left(r^{2}+r_{0}^{2}\right)} I_{0}\left(\frac{2 r_{0} r}{\sigma^{2} \mathcal{L}}\right) \sum_{m=1}^{+\infty} \frac{\beta_{m}}{\sinh \left(\beta_{m}\right)} \\
& \stackrel{(a)}{\geq} \frac{p_{R \mid R_{0}}\left(r \mid r_{0}\right)}{2 \pi}\left(1-\sqrt{2} e^{-\left(\Re\left(a_{1}\right)-\frac{1}{\sigma^{2} \mathcal{L}}\right) r_{0}^{2}} \sum_{m=1}^{+\infty} \frac{\beta_{m}}{\sinh \left(\beta_{m}\right)}\right) \\
& =\frac{1}{2 \pi} p_{R \mid R_{0}}\left(r \mid r_{0}\right)\left(1-\xi\left(r_{0}\right)\right),
\end{aligned}
$$

where $\xi\left(r_{0}\right)$ is defined in (27). Step (a) follows from (4) and

$$
e^{-\left(\Re\left(a_{1}\right)-\frac{1}{\sigma^{2} \mathcal{L}}\right)\left(r^{2}+r_{0}^{2}\right)} \leq e^{-\left(\Re\left(a_{1}\right)-\frac{1}{\sigma^{2} \mathcal{L}}\right) r_{0}^{2}},
$$

which holds because, from (31)-(32), $\Re\left(a_{1}\right)>\frac{1}{\sigma^{2} \mathcal{L}}$. Finally, since $\Re\left(a_{1}\right)>\frac{1}{\sigma^{2} \mathcal{L}}, \xi\left(r_{0}\right) \rightarrow 0$ as $r_{0} \rightarrow+\infty$.

Lemma 5 (Bounds on the Output pdf) Consider the conditional pdf (3). Let $F_{0}\left(r_{0}, \phi_{0}\right) \stackrel{\text { def }}{=} F_{X}\left(r_{0}, \phi_{0}\right)$ be an input cdf and denote by $p\left(r, \phi ; F_{0}\right)$ the corresponding output pdf:

$$
p\left(r, \phi ; F_{0}\right)=\int p\left(r, \phi \mid r_{0}, \phi_{0}\right) d F_{0}\left(r_{0}, \phi_{0}\right) .
$$

1) If $F_{R_{0}}\left(r_{0}\right) \in \mathcal{P}$, then

$$
p\left(r, \phi ; F_{0}\right) \leq \frac{2 k_{u} r}{\sigma^{2} \mathcal{L}} e^{-\frac{r^{2}-2 r \rho}{\sigma^{2} \mathcal{L}}},
$$

where recall that $\rho$ is the peak amplitude defined in (8).

2) If $F_{R_{0}}\left(r_{0}\right) \in \mathcal{A}$, then

$$
p\left(r, \phi ; F_{0}\right) \leq \frac{2 k_{u} r}{\sigma^{2} \mathcal{L}}\left(e^{-\frac{r^{2}}{4 \sigma^{2} \mathcal{L}}}+\frac{A}{\mathcal{C}\left(\frac{r}{2}\right)}\right),
$$

where $k_{u}$ is defined in (23), and recall that $A$ is the average cost defined in (9).

3) If $F_{R_{0}}\left(r_{0}\right) \in \mathcal{F}$, then for large values of $r$

$$
p\left(r, \phi ; F_{0}\right) \geq \frac{k_{1} r}{\pi \sigma^{2} \mathcal{L}} e^{-\frac{r^{2}}{\sigma^{2} \mathcal{L}}}(1-\xi(r)),
$$

where $\xi(\cdot)$ is defined in Lemma 4 and $k_{1}=\int_{0}^{+\infty} e^{-\frac{r_{0}^{2}}{\sigma^{2} \mathcal{L}}} d F_{R_{0}}\left(r_{0}\right)$. Recall that $\mathcal{F}$ is any of the sets $\mathcal{P}, \mathcal{A}$ or $\mathcal{P} \cap \mathcal{A}^{\prime}$ defined in Section III] Furthermore, for $r \geq 0$

$$
p\left(r ; F_{R_{0}}\right) \geq \frac{2 k_{1} r}{\sigma^{2} \mathcal{L}} e^{-\frac{r^{2}}{\sigma^{2} \mathcal{L}}},
$$

where $p\left(r ; F_{R_{0}}\right)=\int p_{R \mid R_{0}}\left(r \mid r_{0}\right) d F_{R_{0}}\left(r_{0}\right)$ and 
Proof: We have

$$
\begin{aligned}
p\left(r, \phi ; F_{0}\right) & =\int p\left(r, \phi \mid r_{0}, \phi_{0}\right) d F_{0}\left(r_{0}, \phi_{0}\right) \\
& \leq k_{u} \int p_{R \mid R_{0}}\left(r \mid r_{0}\right) d F_{R_{0}}\left(r_{0}\right) \\
& =k_{u} p\left(r ; F_{R_{0}}\right),
\end{aligned}
$$

where we used the upper bound (22) in Lemma 4. We bound $p\left(r ; F_{R_{0}}\right)$ for the three cases below.

Case 1) $F_{R_{0}}\left(r_{0}\right) \in \mathcal{P}$. We have

$$
\begin{aligned}
p\left(r ; F_{R_{0}}\right) & =\int p_{R \mid R_{0}}\left(r \mid r_{0}\right) d F_{R_{0}}\left(r_{0}\right) \\
& =\frac{2 r}{\sigma^{2} \mathcal{L}} \int_{0}^{\rho} e^{-\frac{r^{2}+r_{0}^{2}}{\sigma^{2} \mathcal{L}}} I_{0}\left(\frac{2 r r_{0}}{\sigma^{2} \mathcal{L}}\right) d F_{R_{0}}\left(r_{0}\right) \\
& \leq \frac{2 r}{\sigma^{2} \mathcal{L}} e^{-\frac{r^{2}-2 r \rho}{\sigma^{2} \mathcal{L}}},
\end{aligned}
$$

where $\rho$ is the peak amplitude in (8) and we used the inequality in Lemma 3 2 , as well as the inequality $\left(r-r_{0}\right)^{2} \geq r^{2}-2 r \rho$ when $r_{0} \in[0, \rho]$.

Case 2) $F_{R_{0}}\left(r_{0}\right) \in \mathcal{A}$. The average cost constraint upper bounds the tail of the input distribution as follows. For any $a \geq 0$

$$
\begin{aligned}
A & \geq \int_{0}^{+\infty} \mathcal{C}\left(r_{0}\right) d F_{R_{0}}\left(r_{0}\right) \\
& \geq \int_{a}^{+\infty} \mathcal{C}\left(r_{0}\right) d F_{R_{0}}\left(r_{0}\right) \\
& \geq \mathcal{C}(a) \int_{a}^{+\infty} d F_{R_{0}}\left(r_{0}\right),
\end{aligned}
$$

where we used the properties C1-C2 of the cost function $\mathcal{C}\left(r_{0}\right)$ in Section III. Therefore

$$
\int_{a}^{+\infty} d F_{R_{0}}\left(r_{0}\right) \leq \frac{A}{\mathcal{C}(a)} .
$$

Note that, since $\mathcal{C}(a)=\omega\left(a^{2}\right), \mathcal{C}(a)$ grows faster than $a^{2}$ as $a \rightarrow \infty$.

We have:

$$
\begin{aligned}
p\left(r ; F_{R_{0}}\right) & =\frac{2 r}{\sigma^{2} \mathcal{L}} \int_{0}^{+\infty} e^{-\frac{r^{2}+r_{0}^{2}}{\sigma^{2} \mathcal{L}}} I_{0}\left(\frac{2 r r_{0}}{\sigma^{2} \mathcal{L}}\right) d F_{R_{0}}\left(r_{0}\right) \\
& \stackrel{(a)}{\leq} \frac{2 r}{\sigma^{2} \mathcal{L}} \int_{0}^{+\infty} e^{-\frac{\left(r-r_{0}\right)^{2}}{\sigma^{2} \mathcal{L}}} d F_{R_{0}}\left(r_{0}\right) \\
& =\frac{2 r}{\sigma^{2} \mathcal{L}}\left(\int_{0}^{\frac{r}{2}} e^{-\frac{\left(r-r_{0}\right)^{2}}{\sigma^{2} \mathcal{L}}} d F_{R_{0}}\left(r_{0}\right)+\int_{\frac{r}{2}}^{+\infty} e^{-\frac{\left(r-r_{0}\right)^{2}}{\sigma^{2} \mathcal{L}}} d F_{R_{0}}\left(r_{0}\right)\right) \\
& \stackrel{(b)}{\leq} \frac{2 r}{\sigma^{2} \mathcal{L}}\left(e^{-\frac{r^{2}}{4 \sigma^{2} \mathcal{L}}}+\int_{\frac{r}{2}}^{+\infty} d F_{R_{0}}\left(r_{0}\right)\right) \\
& \leq \frac{2 r}{\sigma^{2} \mathcal{L}}\left(e^{-\frac{r^{2}}{4 \sigma^{2} \mathcal{L}}}+\frac{A}{\mathcal{C}\left(\frac{r}{2}\right)}\right)
\end{aligned}
$$

Step $(a)$ follows from Lemma 3- 2. Step $(b)$ follows because $\exp \left(-\frac{\left(r-r_{0}\right)^{2}}{\sigma^{2} \mathcal{L}}\right)$ increases with $r_{0} \in\left[0, \frac{r}{2}\right]$, and exp $\left(-\frac{\left(r-r_{0}\right)^{2}}{\sigma^{2} \mathcal{L}}\right) \leq$ 1. Step (c) follows from (40) with $a=r / 2$. 
Case 3) We have for large values of $r$

$$
\begin{aligned}
p\left(r, \phi ; F_{0}\right) & =\int p\left(r, \phi \mid r_{0}, \phi_{0}\right) d F_{0}\left(r_{0}, \phi_{0}\right) \\
& \stackrel{(a)}{\geq} \int\left(\frac{1}{2 \pi} p_{R \mid R_{0}}\left(r \mid r_{0}\right)-\frac{1}{\pi} \frac{\sqrt{2} r}{\sigma^{2} \mathcal{L}} e^{-\Re\left(a_{1}\right)\left(r^{2}+r_{0}^{2}\right)} I_{0}\left(\frac{2 r_{0} r}{\sigma^{2} \mathcal{L}}\right) \sum_{m=1}^{+\infty} \frac{\beta_{m}}{\sinh \left(\beta_{m}\right)}\right) d F_{R_{0}}\left(r_{0}\right) \\
& \stackrel{(b)}{=} \frac{r}{\pi \sigma^{2} \mathcal{L}} e^{-\frac{r^{2}}{\sigma^{2} \mathcal{L}}} \int I_{0}\left(\frac{2 r_{0} r}{\sigma^{2} \mathcal{L}}\right) e^{-\frac{r_{0}^{2}}{\sigma^{2} \mathcal{L}}}\left(1-\sqrt{2} e^{-\left(\Re\left(a_{1}\right)-\frac{1}{\sigma^{2} \mathcal{L}}\right)\left(r^{2}+r_{0}^{2}\right)} \sum_{m=1}^{+\infty} \frac{\beta_{m}}{\sinh \left(\beta_{m}\right)}\right) d F_{R_{0}}\left(r_{0}\right) \\
& \stackrel{(c)}{\geq} \frac{r}{\pi \sigma^{2} \mathcal{L}} e^{-\frac{r^{2}}{\sigma^{2} \mathcal{L}}}\left(1-\sqrt{2} e^{-\left(\Re\left(a_{1}\right)-\frac{1}{\sigma^{2} \mathcal{L}}\right) r^{2}} \sum_{m=1}^{+\infty} \frac{\beta_{m}}{\sinh \left(\beta_{m}\right)}\right) \int I_{0}\left(\frac{2 r_{0} r}{\sigma^{2} \mathcal{L}}\right) e^{-\frac{r_{0}^{2}}{\sigma^{2} \mathcal{L}}} d F_{R_{0}}\left(r_{0}\right) \\
& \stackrel{(d)}{\geq} \frac{k_{1} r}{\pi \sigma^{2} \mathcal{L}} e^{-\frac{r^{2}}{\sigma^{2} \mathcal{L}}}(1-\xi(r)),
\end{aligned}
$$

where $\xi(\cdot)$ is defined in Lemma 4 and $k_{1}$ is defined in the statement of the lemma. Step (a) follows from (37) and step $(b)$ follows from (4). Step $(c)$ holds true since $e^{-\left(\Re\left(a_{1}\right)-\frac{1}{\sigma^{2} \mathcal{L}}\right) r_{0}^{2}}<1$ which is true by virtue of (32). Finally, step $(d)$ is justified by virtue of Lemma 3 . 1 and the fact that $\xi(r) \rightarrow 0$ as $r \rightarrow+\infty$. Also we have for $r \geq 0$

$$
\begin{aligned}
p\left(r ; F_{R_{0}}\right) & =\int_{0}^{+\infty} p_{R \mid R_{0}}\left(r \mid r_{0}\right) d F_{R_{0}}\left(r_{0}\right) \\
& \stackrel{(a)}{=} \frac{2 r}{\sigma^{2} \mathcal{L}} e^{-\frac{r^{2}}{\sigma^{2} \mathcal{L}}} \int_{0}^{+\infty} e^{-\frac{r_{0}^{2}}{\sigma^{2} \mathcal{L}}} I_{0}\left(\frac{2 r r_{0}}{\sigma^{2} \mathcal{L}}\right) d F_{R_{0}}\left(r_{0}\right) \\
& \stackrel{(b)}{\geq} \frac{2 k_{1} r}{\sigma^{2} \mathcal{L}} e^{-\frac{r^{2}}{\sigma^{2} \mathcal{L}}}
\end{aligned}
$$

Step $(a)$ follows from (4). Step $(b)$ follows from Lemma 3 . 1.

Define the conditional entropy density

$$
i\left(r, \phi, r_{0}, \phi_{0}\right) \stackrel{\text { def }}{=} \begin{cases}-p\left(r, \phi \mid r_{0}, \phi_{0}\right) \ln p\left(r, \phi \mid r_{0}, \phi_{0}\right), & \text { if } p\left(r, \phi \mid r_{0}, \phi_{0}\right)>0 \\ 0, & \text { if } p\left(r, \phi \mid r_{0}, \phi_{0}\right)=0\end{cases}
$$

Let $F_{0}\left(r_{0}, \phi_{0}\right) \stackrel{\text { def }}{=} F_{X}\left(r_{0}, \phi_{0}\right)$ be an input cdf and denote by $p\left(r, \phi ; F_{0}\right)$ the corresponding output pdf, i.e.,

$$
p\left(r, \phi ; F_{0}\right)=\int p\left(r, \phi \mid r_{0}, \phi_{0}\right) d F_{0}\left(r_{0}, \phi_{0}\right) .
$$

We prove that $i\left(r, \phi, r_{0}, \phi_{0}\right)$ is continuous, bounded and its average is upper bounded by an integrable function.

Lemma 6 We have:

1) $i\left(r, \phi, r_{0}, \phi_{0}\right)$ is continuous and bounded in $\left(r, \phi, r_{0}, \phi_{0}\right)$, for all $r, r_{0} \geq 0$ and $\phi, \phi_{0} \in[0,2 \pi)$.

2) For any $F_{R_{0}}\left(r_{0}\right) \in \mathcal{F}$,

$$
\left|\int i\left(r, \phi, r_{0}, \phi_{0}\right) d F_{0}\left(r_{0}, \phi_{0}\right)\right| \leq d(r, \phi),
$$

where $d(r, \phi)$ is independent of $F_{0}\left(r_{0}, \phi_{0}\right)$ and $\int_{r, \phi} d(r, \phi) d r d \phi<\infty$.

Proof: The continuity of $i\left(r, \phi, r_{0}, \phi_{0}\right)$ follows from the definition of the conditional pdf (3). The boundedness is due to upper and lower bounds on $p\left(r, \phi \mid r_{0}, \phi_{0}\right)$ established in Lemma 4 . This completes Part 1) of the lemma.

For Part 2), since $p \ln (p)<0$ when $p \in(0,1)$, we break down the integral into two parts:

$$
\left|\int i\left(r, \phi, r_{0}, \phi_{0}\right) d F_{0}\left(r_{0}, \phi_{0}\right)\right| \leq I^{+}(r, \phi)+I^{-}(r, \phi),
$$

where

$$
\begin{aligned}
& I^{+}(r, \phi) \stackrel{\text { def }}{=} \int_{p\left(r, \phi \mid r_{0}, \phi_{0}\right) \leq 1} i\left(r, \phi, r_{0}, \phi_{0}\right) d F_{0}\left(r_{0}, \phi_{0}\right), \\
& I^{-}(r, \phi) \stackrel{\text { def }}{=}-\int_{p\left(r, \phi \mid r_{0}, \phi_{0}\right)>1} i\left(r, \phi, r_{0}, \phi_{0}\right) d F_{0}\left(r_{0}, \phi_{0}\right) .
\end{aligned}
$$

We upper bound $I^{ \pm}(r, \phi)$ for the peak amplitude and average cost constraints separately. The upper bound on $I^{-}(r, \phi)$ is based on the upper bound on the conditional pdf in Lemma 4 . The upper bound on $I^{+}(r, \phi)$ is based on the lower bound on the conditional pdf in Lemma 4.2. 
Case 1) Peak amplitude constraint.: Suppose that $F_{0}\left(r_{0}, \phi_{0}\right)$ is subjected to a peak amplitude constraint, i.e., $F_{R_{0}}\left(r_{0}\right) \in \mathcal{P}$. From the lower bound (26) in Lemma 4, there exists a finite $c>0$ for which $\xi(c)<\frac{1}{2}$ such that

$$
\begin{aligned}
\ln \frac{1}{p\left(r, \phi \mid c, \phi_{0}\right)} & \leq \ln \frac{1}{\frac{1}{2 \pi} p_{R \mid R_{0}}(r \mid c)(1-\xi(c))} \\
& \leq \ln \frac{4 \pi}{p_{R \mid R_{0}}(r \mid c)} .
\end{aligned}
$$

On the other hand, choosing $c$ large enough and finite, we obtain

$$
\ln \frac{1}{p\left(r, \phi \mid r_{0}, \phi_{0}\right)} \leq \ln \frac{1}{p\left(r, \phi \mid c, \phi_{0}\right)},
$$

for $0 \leq r_{0} \leq \rho$. This holds true since $p\left(r, \phi \mid c, \phi_{0}\right) \rightarrow 0$ as $c \rightarrow+\infty$.

Inequalities (42) and (43) imply

$$
\begin{aligned}
I^{+}(r, \phi) & \leq \int_{0<p\left(r, \phi \mid r_{0}, \phi_{0}\right) \leq 1} p\left(r, \phi \mid r_{0}, \phi_{0}\right) \ln \frac{4 \pi}{p_{R \mid R_{0}}(r \mid c)} d F_{0}\left(r_{0}, \phi_{0}\right) \\
& \leq\left(\ln (4 \pi)-\ln p_{R \mid R_{0}}(r \mid c)\right) p\left(r, \phi ; F_{0}\right) \\
& \leq\left(\ln (4 \pi)-\ln \frac{2 r}{\sigma^{2} \mathcal{L}}+\frac{r^{2}+c^{2}}{\sigma^{2} \mathcal{L}}\right) p\left(r, \phi ; F_{0}\right),
\end{aligned}
$$

where we used the bound $p_{R \mid R_{0}}(r \mid c) \geq \frac{2 r}{\sigma^{2} \mathcal{L}} e^{-\frac{r^{2}+c^{2}}{\sigma^{2} \mathcal{L}}}$.

Similarly, for $I^{-}(r, \phi)$ we have

$$
\begin{aligned}
I^{-}(r, \phi) & \leq \int_{p\left(r, \phi \mid r_{0}, \phi_{0}\right)>1} p\left(r, \phi \mid r_{0}, \phi_{0}\right) \ln k_{u} p_{R \mid R_{0}}\left(r \mid r_{0}\right) d F_{0}\left(r_{0}, \phi_{0}\right) \\
& \leq\left(\ln k_{u}+\ln \frac{2 r}{\sigma^{2} \mathcal{L}}\right) p\left(r, \phi ; F_{0}\right),
\end{aligned}
$$

where we used Lemma 44 in (45) and (46) follows from (25).

Finally, adding (44) and (46)

$$
\begin{aligned}
I^{+}(r, \phi)+I^{-}(r, \phi) & \leq\left(\ln (4 \pi)-\ln \frac{2 r}{\sigma^{2} \mathcal{L}}+\frac{r^{2}+c^{2}}{\sigma^{2} \mathcal{L}}\right) p\left(r, \phi ; F_{0}\right)+\left(\ln k_{u}+\ln \frac{2 r}{\sigma^{2} \mathcal{L}}\right) p\left(r, \phi ; F_{0}\right) \\
& \leq\left(\ln \left(4 \pi k_{u}\right)+\frac{r^{2}+c^{2}}{\sigma^{2} \mathcal{L}}\right) \frac{2 k_{u} r}{\sigma^{2} \mathcal{L}} e^{-\frac{r^{2}-2 r \rho}{\sigma^{2} \mathcal{L}}} \\
& \stackrel{\text { def }}{=} d(r, \phi),
\end{aligned}
$$

where we used Lemma 5 - 1 to obtain (47). Clearly, $d(r, \phi)$ is is an integrable function of $(r, \phi)$.

Case 2) Average cost constraint: Suppose that $F_{0}\left(r_{0}, \phi_{0}\right)$ is subject to an average cost constraint, i.e., $F_{R_{0}}\left(r_{0}\right) \in \mathcal{A}$. The inequality (46) holds true and

$$
\begin{aligned}
I^{-}(r, \phi) & \leq\left(\ln k_{u}+\ln \frac{2 r}{\sigma^{2} \mathcal{L}}\right) p\left(r, \phi ; F_{0}\right) \\
& \leq\left(\ln k_{u}+\ln \frac{2 r}{\sigma^{2} \mathcal{L}}\right) \frac{2 k_{u} r}{\sigma^{2} \mathcal{L}}\left(e^{-\frac{r^{2}}{4 \sigma^{2} \mathcal{L}}}+\frac{A}{\mathcal{C}\left(\frac{r}{2}\right)}\right),
\end{aligned}
$$

where we used Lemma 55 2 in obtaining the last inequality. The right hand side of (48) is integrable because $\mathcal{C}(r)=\omega\left(r^{2}\right)$.

Finally, we obtain an upper bound on $I^{+}(r, \phi)$. We apply the inequality

$$
|x \ln x| \leq \frac{1}{1-\delta} x^{\delta}
$$


which is valid for all $0<x \leq 1$ and $0<\delta<1$. We obtain

$$
\begin{aligned}
I^{+}(r, \phi) & =-\int_{0<p\left(r, \phi \mid r_{0}, \phi_{0}\right) \leq 1} p\left(r, \phi \mid r_{0}, \phi_{0}\right) \ln p\left(r, \phi \mid r_{0}, \phi_{0}\right) d F\left(r_{0}, \phi_{0}\right) \\
& \leq \frac{1}{1-\delta} \int_{0<p\left(r, \phi \mid r_{0}, \phi_{0}\right) \leq 1}\left(p\left(r, \phi \mid r_{0}, \phi_{0}\right)\right)^{\delta} d F\left(r_{0}, \phi_{0}\right) \\
& \leq \frac{1}{1-\delta} k_{u}^{\delta}\left(p_{R \mid R_{0}}\left(r \mid r_{0}\right)\right)^{\delta} d F\left(r_{0}\right) \\
& \leq \frac{1}{1-\delta} k_{u}^{\delta}\left(\frac{2 r}{\sigma^{2} \mathcal{L}}\right)^{\delta} \int e^{-\delta \frac{\left(r-r_{0}\right)^{2}}{\sigma^{2} \mathcal{L}}} d F\left(r_{0}\right) \\
& \leq \frac{1}{1-\delta}\left(\frac{2 k_{u} r}{\sigma^{2} \mathcal{L}}\right)^{\delta}\left(e^{-\frac{\delta r^{2}}{4 \sigma^{2} \mathcal{L}}}+\frac{A}{\mathcal{C}\left(\frac{r}{2}\right)}\right)
\end{aligned}
$$

The inequality (50) follows from the upper bound in Lemma 4. Inequality (51) follows from (24). The last inequality can be justified using the same set of inequalities that yielded inequality (38). Clearly, the right hand side in (52) is integrable because $\mathcal{C}(r)=\omega\left(r^{2}\right)$.

The inequalities (48) and (52) prove the desired inequality in the Part 2) of the lemma.

Lemma 7 (Continuity of the Conditional Entropy) Let $F_{X}\left(r_{0}, \phi_{0}\right)$ be an input cdf such that $F_{R_{0}}\left(r_{0}\right) \in \mathcal{F}$. The conditional entropy $h(Y \mid X)$ in the channel (3) is continuous function of $F_{X}$.

Proof:

Let $\left\{X_{m}=\left(R_{m}, \Phi_{m}\right)\right\}_{m \geq 1}$ be a sequence of input variables whose cdfs are denoted respectively by $\left\{F_{m}\left(r_{0}, \phi_{0}\right)\right\}_{m \geq 1}$ and such that $F_{R_{m}}\left(r_{0}\right) \in \mathcal{F}$. Suppose that $\left\{F_{m}\left(r_{0}, \phi_{0}\right)\right\}_{m \geq 1}$ converges weakly to $F_{X}\left(r_{0}, \phi_{0}\right) \stackrel{\text { def }}{=} F_{0}\left(r_{0}, \phi_{0}\right)$. We have

$$
\begin{aligned}
h(Y \mid X) & =\int h\left(Y \mid r_{0}, \phi_{0}\right) d F_{0}\left(r_{0}, \phi_{0}\right) \\
& =-\iint_{r, \phi} p\left(r, \phi \mid r_{0}, \phi_{0}\right) \ln p\left(r, \phi \mid r_{0}, \phi_{0}\right) d r d \phi d F_{0}\left(r_{0}, \phi_{0}\right) \\
& =\int_{r, \phi} \int i\left(r, \phi, r_{0}, \phi_{0}\right) d F_{0}\left(r_{0}, \phi_{0}\right) d r d \phi \\
& =\int_{r, \phi} \lim _{m \rightarrow+\infty} \int i\left(r, \phi, r_{0}, \phi_{0}\right) d F_{m}\left(r_{0}, \phi_{0}\right) d r d \phi \\
& =\lim _{m \rightarrow+\infty} \int_{r, \phi} \int i\left(r, \phi, r_{0}, \phi_{0}\right) d F_{m}\left(r_{0}, \phi_{0}\right) d r d \phi \\
& =\lim _{m \rightarrow+\infty} \iint_{r, \phi} i\left(r, \phi, r_{0}, \phi_{0}\right) d r d \phi d F_{m}\left(r_{0}, \phi_{0}\right) \\
& =\lim _{m \rightarrow+\infty} h\left(Y \mid X_{m}\right) .
\end{aligned}
$$

The order of the integrals in (53) and (56) can be exchanged by applying the Fubini's theorem and using Lemma 6 . 2. Equation (54) is due to the weak convergence and to the fact that $i\left(r, \phi, r_{0}, \phi_{0}\right)$ is continuous and bounded using Lemma 6 . 1 . The order of the limit and the integral in 54-(55) can be exchanged by applying the dominated convergence theorem and using Lemma 6.2.

Lemma 8 (Continuity of the Output Entropy) The output entropy $h(Y)$ in the channel (3) is continuous function of the input cdf $F_{X}\left(r_{0}, \phi_{0}\right)$, where $F_{R_{0}}\left(r_{0}\right) \in \mathcal{F}$.

Proof:

Let $F_{X}\left(r_{0}, \phi_{0}\right) \stackrel{\text { def }}{=} F_{0}\left(r_{0}, \phi_{0}\right)$ be such that $F_{R_{0}}\left(r_{0}\right) \in \mathcal{F}$ and let $p\left(r, \phi ; F_{0}\right)=\int p\left(r, \phi \mid r_{0}, \phi_{0}\right) d F_{0}\left(r_{0}, \phi_{0}\right)$ be the corresponding output pdf in the channel (3). From (3), $p\left(r, \phi \mid r_{0}, \phi_{0}\right)$ is a continuous bounded function of $\left(r_{0}, \phi_{0}\right)$. Thus, from the definition of the weak convergence, $p\left(r, \phi ; F_{0}\right)$ is continuous in $F_{0}$. Hence $p\left(r, \phi ; F_{0}\right) \mid \ln \left(p\left(r, \phi ; F_{0}\right) \mid\right.$ is continuous in $F_{0}$. Second, from Lemma 5 $3, p\left(r, \phi ; F_{0}\right) \mid \ln \left(p\left(r, \phi ; F_{0}\right) \mid\right.$ is dominated by an integrable function of $(r, \phi)$, that is independent of $F_{0}$. Hence $h(Y)$ is continuous in output pdf. Combining these two results proves the lemma. 


\section{APPENDIX II \\ AnALYTiCity OF THE KKT CONDitions}

Consider $\operatorname{LHS}_{\rho}\left(r_{0}\right)$ and $\mathrm{LHS}_{A}\left(r_{0}\right)$ in the KKT conditions defined in (12) and (13) respectively.

Lemma 9 (Analytic Extensions of $\mathbf{L H S}_{\rho}\left(r_{0}\right)$ and $\mathbf{L H S}_{A}\left(r_{0}\right)$ ) There exists a $\delta>0$ and a non-empty open connected region $\mathcal{O}_{\delta}$ in the complex plane containing the non-negative real line $\mathbb{R}_{0}^{+}=\{z \in \mathbb{R}: z \geq 0\}$ such that LHS $S_{\rho}\left(r_{0}\right)$ and LHS ${ }_{A}\left(r_{0}\right)$ can be analytically extended from $r_{0} \in \mathbb{R}_{0}^{+}$to $z \in \mathcal{O}_{\delta}$.

Proof:

We break down the proof into five steps.

1) Analyticity of $p\left(r, \phi \mid z, \phi_{0}\right)$ : Let

$$
s\left(z ; r, \phi, \phi_{0}\right)=\frac{1}{2 \pi}\left(p_{R \mid R_{0}}(r \mid z)+\sum_{m=-\infty, m \neq 0}^{+\infty} C_{m}(r, z) e^{j m\left(\phi-\phi_{0}-\gamma z^{2} \mathcal{L}\right)}\right),
$$

be an extension of $p\left(r, \phi \mid r_{0}, \phi_{0}\right)$ defined by (3) from $r_{0} \in \mathbb{R}_{0}^{+}$to the complex plane $r_{0} \in \mathbb{C}$. We note that after straightforward manipulations of equations (5), (6), and (7), it can be shown that $a_{-m}=a_{m}^{*}, b_{-m}=b_{m}^{*}$ and $C_{-m}\left(r, r_{0}\right)=C_{m}^{*}\left(r, r_{0}\right), m \in \mathbb{N}$. We prove that $s\left(z ; r, \phi, \phi_{0}\right)$ is an entire function.

From Lemma 3-6, $I_{m}(z)$ is an entire function of $z$ for $m \in \mathbb{Z}$. Thus, $p_{R \mid R_{0}}(r \mid z)$ and each term in the sum in (57) are entire functions. Below, we show that $\left|C_{m}(r, z) e^{j m\left(\phi-\phi_{0}-\gamma z^{2} \mathcal{L}\right)}\right|$ can be upper bounded in $\mathbb{C}$ for large values of $m$ by an absolutely summable sequence of $m$ that is independent of $z$. Thus the series (57) converges uniformly over $z \in \mathbb{C}$. The sum of a uniformly convergent sequence of analytic functions is analytic. Therefore, $s\left(z ; r, \phi, \phi_{0}\right)$ is an analytic function on $\mathbb{C}$.

We have

$$
\begin{aligned}
\mid C_{m}(r, z) e^{j m\left(\phi-\phi_{0}-\gamma z^{2} \mathcal{L}\right) \mid} & =\left|r b_{m} e^{-a_{m}\left(r^{2}+z^{2}\right)} I_{m}\left(2 b_{m} z r\right) e^{-j m \gamma z^{2} \mathcal{L}}\right| \\
& =r\left|b_{|m|}\right|\left|I_{m}\left(2 b_{m} z r\right)\right| e^{-\Re\left(a_{m}\right) r^{2}} e^{-\Re\left(a_{m}\right) \Re\left(z^{2}\right)} e^{\left(\Im\left(a_{m}\right)+m \gamma \mathcal{L}\right) \Im\left(z^{2}\right)},
\end{aligned}
$$

where we used the fact that $\left|b_{m}\right|=\left|b_{|m|}\right|$ since $b_{-m}=b_{m}^{*}$. Recall from the proof of Lemma 4 that $\Re\left(a_{m}\right) \stackrel{\text { def }}{=} \frac{1}{\sigma^{2} \mathcal{L}} t\left(\beta_{m}\right)$ where $t(x)$ is given by equation (30) and $\beta_{m} \stackrel{\text { def }}{=} \sqrt{\frac{m \gamma}{2}} \sigma \mathcal{L}, m>0$. Also, the imaginary part of $a_{m}$ in $(6)$ is $\Im\left(a_{m}\right) \stackrel{\text { def }}{=} \frac{1}{\sigma^{2} \mathcal{L}} \tau\left(\beta_{m}\right)$, where

$$
\tau(x)=\frac{x(\sinh (2 x)-\sin (2 x))}{2\left(\sinh ^{2}(x)+\sin ^{2}(x)\right)} .
$$

Notice that $t(x) \equiv x$ and $\tau(x) \equiv x$ as $x \rightarrow+\infty$. Let $\epsilon>0$, then there exists an $M>0$ such that whenever $|m|>M$, we have

$$
\begin{aligned}
& 0<(1-\epsilon) \frac{\beta_{|m|}}{\sigma^{2} \mathcal{L}}<\Re\left(a_{m}\right)<(1+\epsilon) \frac{\beta_{|m|}}{\sigma^{2} \mathcal{L}} \\
& 0<(1-\epsilon) \frac{\beta_{|m|}}{\sigma^{2} \mathcal{L}}<\left|\Im\left(a_{m}\right)\right|<(1+\epsilon) \frac{\beta_{|m|}}{\sigma^{2} \mathcal{L}},
\end{aligned}
$$

where we used the fact that $a_{-m}=a_{m}^{*}$. Now, let $\mathcal{B}\left(z_{0}, \zeta\right)=\left\{z:\left|z-z_{0}\right|<\zeta\right\}$ be a neighborhood of $z_{0} \in \mathbb{C}$. If $z \in \mathcal{B}\left(z_{0}, \zeta\right)$, then $\left|z^{2}\right|<\left(\left|z_{0}\right|+\zeta\right)^{2}$ and

$$
\begin{aligned}
& -\Re\left(a_{m}\right) \Re\left(z^{2}\right)<(1+\epsilon) \frac{\beta_{|m|}}{\sigma^{2} \mathcal{L}}\left(\left|z_{0}\right|+\zeta\right)^{2} \\
& \Im\left(a_{m}\right) \Im\left(z^{2}\right)<(1+\epsilon) \frac{\beta_{|m|}}{\sigma^{2} \mathcal{L}}\left(\left|z_{0}\right|+\zeta\right)^{2} \\
& -\Re\left(a_{m}\right) r^{2}<-\frac{r^{2}}{\sigma^{2} \mathcal{L}},
\end{aligned}
$$

for $|m|>M$, where we used inequalities (59), (60), and (32). Also, for $|m|$ large enough, we have $\left|b_{|m|}\right|<1$ as $|m| \rightarrow+\infty$ as inferred by equation (34) and

$$
\left|I_{m}\left(2 b_{m} z r\right)\right|=\left|I_{|m|}\left(2 b_{m} z r\right)\right| \leq(1+\epsilon) \frac{\left(2\left|b_{m}\right||z| r\right)^{|m|}}{|m| !} \leq(1+\epsilon) \frac{\left(2\left(\left|z_{0}\right|+\zeta\right) r\right)^{|m|}}{|m| !}
$$


where we used Lemma 3.3. Using the bounds in (61), 62), (63), and (64), we obtain from (58) for $|m|>M$

$$
\begin{aligned}
& \left|C_{m}(r, z) e^{j m\left(\phi-\phi_{0}-\gamma z^{2} \mathcal{L}\right)}\right| \leq(1+\epsilon) r e^{-\frac{r^{2}}{\sigma^{2} \mathcal{L}}} \frac{\left(2\left(\left|z_{0}\right|+\zeta\right)\right)^{|m|} r^{|m|}}{|m| !} e^{2(1+\epsilon) \frac{\beta_{|m|}\left(\left|z_{0}\right|+\zeta\right)^{2}}{\sigma^{2} \mathcal{L}}} e^{|m| \gamma \mathcal{L}\left(\left|z_{0}\right|+\zeta\right)^{2}} \\
& \leq(1+\epsilon) r e^{-\frac{r^{2}}{\sigma^{2} \mathcal{L}}} \frac{\left(2 r\left(\left|z_{0}\right|+\zeta\right) e^{\sqrt{2 \gamma}(1+\epsilon) \frac{\left(\left|z_{0}\right|+\zeta\right)^{2}}{\sigma}} e^{\gamma \mathcal{L}\left(\left|z_{0}\right|+\zeta\right)^{2}}\right)^{|m|}}{|m| !},
\end{aligned}
$$

where we replaced $\beta_{|m|}$ by its expression and we used the fact that $\sqrt{|m|}<|m|$ for large values of $|m|$. It can be seen that the z-independent upper bound in (65) is summable over $m$. Thus the infinite sum in (57) is uniformly convergent for all $z \in \mathbb{C}$ and $s\left(z ; r, \phi, \phi_{0}\right)$ is an entire function.

2) Analyticity of $s\left(z ; r, \phi, \phi_{0}\right) \ln \left(s\left(z ; r, \phi, \phi_{0}\right)\right)$ : We show that there exists an open covering $\mathcal{O}$ of $\mathbb{R}_{0}^{+}$such that the function $s\left(z ; r, \phi, \phi_{0}\right) \ln \left(s\left(z ; r, \phi, \phi_{0}\right)\right)$ is analytic in $z \in \mathcal{O}$. We proved in the previous part that $s\left(z ; r, \phi, \phi_{0}\right)$ is analytic in $z \in \mathbb{C}$. As for the analyticity of $s\left(z ; r, \phi, \phi_{0}\right) \ln \left(s\left(z ; r, \phi, \phi_{0}\right)\right)$ :

- We have

$$
\begin{aligned}
p\left(r, \phi \mid r_{0}, \phi_{0}\right) & =p\left(r \mid r_{0}, \phi_{0}\right) p\left(\phi \mid r, r_{0}, \phi_{0}\right) \\
& =p\left(r \mid r_{0}\right) p\left(\phi \mid r, r_{0}, \phi_{0}\right),
\end{aligned}
$$

where (66) follows because $p\left(r \mid r_{0}, \phi_{0}\right)=p\left(r \mid r_{0}\right)$ from (2). From (3), $p\left(r \mid r_{0}\right)>0$ for $r \in(0, \infty)$ and $r_{0} \geq 0$. For the phase conditional pdf in (66), we apply the Karhunen-Loéve expansion in [3, Sec. V], to write the phase $\Phi$ as $\Phi_{0}$ plus a weighted sum $S$ of $M \rightarrow \infty$ independent non-central chi-squared random variables with two degrees-of-freedom and a centrality parameter depending on $r_{0}$. The $\operatorname{PDF} p_{S}(s ; M)$ is a generalized chi-squared random variable. It can be verified that $p_{S}(s ; M)>c$ for $s \in(0, \infty)$, where $c$ is independent of $M$, so that $p_{S}(s ; \infty)>0$. The $p\left(\phi \mid r_{0}, \phi_{0}\right)$ is the wrapped distribution of $p_{S}(s ; \infty)$, thus $p\left(\phi \mid \phi_{0}, r_{0}\right)>0, r_{0} \in[0, \infty), \forall \phi_{0} \in[0,2 \pi)$. Finally, using [23, Eq. 7], fixing $R$ fixes only one term in $S$, leaving $M-1$ independent terms. Thus $p\left(\phi \mid \phi_{0}, r_{0}, r\right)>0$. Summarizing, if $r \in(0, \infty)$, then

$$
p\left(r, \phi \mid r_{0}, \phi_{0}\right)>0, \quad \forall r_{0} \in[0, \infty), \quad \forall \phi_{0} .
$$

- For any $z_{l} \in \mathbb{R}_{0}^{+}$, there exits an open ball $\mathcal{B}_{l}\left(z_{l}, \zeta_{l}\right)$ such that $\Re\left(s\left(z ; r, \phi, \phi_{0}\right)\right)>0$, when $z \in \mathcal{B}_{l}$. This is true because $s\left(r_{0} ; r, \phi, \phi_{0}\right)=p\left(r, \phi \mid r_{0}, \phi_{0}\right)$ is positive in $0 \leq r_{0}<\infty$ (up to a set of Lebesgue measure zero) and continuous on $\mathbb{C}$.

- Therefore, there exists a sequence $\left\{z_{i}\right\}_{i \geq 1}$ of real non-negative numbers and an open covering $\left\{\mathcal{O}=\cup_{i \geq 1} \mathcal{B}_{i}\right\} \subset \mathbb{C}$ of the non-negative real line such that $\Re\left(s\left(z ; r, \phi, \phi_{0}\right)\right)>0$.

- Taking the principal branch of the $\operatorname{logarithm}, \log (z)$ is analytic in $\mathbb{C} \backslash \mathbb{R}_{0}^{+}$. Since $\Re\left(s\left(z ; r, \phi, \phi_{0}\right)\right)>0$, we obtain that $s\left(z ; r, \phi, \phi_{0}\right) \ln \left(s\left(z ; r, \phi, \phi_{0}\right)\right)$ is analytic in $z \in \mathcal{O}$.

It follows that $s\left(z ; r, \phi, \phi_{0}\right) \ln \left(s\left(z ; r, \phi, \phi_{0}\right)\right)$ is analytic in $z \in \mathcal{O}$.

3) Analyticity of $\int_{0}^{2 \pi} h\left(R, \Phi \mid z, \phi_{0}\right) d \phi_{0}$ : We now prove that

$$
\begin{aligned}
u(z) & =\int_{0}^{2 \pi} h\left(R, \Phi \mid z, \phi_{0}\right) d \phi_{0} \\
& =\int_{0}^{2 \pi} \int_{0}^{2 \pi} \int_{0}^{+\infty} s\left(z ; r, \phi, \phi_{0}\right) \ln \left(s\left(z ; r, \phi, \phi_{0}\right)\right) d r d \phi d \phi_{0},
\end{aligned}
$$

is analytic in $z \in \mathcal{O}$. The proof is based on the Morera's theorem.

Morera's Theorem [21]. If $f(z)$ is continuous in an open region $\mathcal{O} \subseteq \mathbb{C}$ and $\int_{\gamma} f(z) d z=0$ for any closed triangular contour $\gamma$ in $\mathcal{O}$, then $f(z)$ is analytic on $\mathcal{O}$.

We first show that $u(z)$ is continuous in $z \in \mathcal{O}$. If $z_{0} \in \mathcal{O}$, then

$$
\begin{aligned}
\lim _{z \rightarrow z_{0}} u(z) & =\lim _{z \rightarrow z_{0}} \int_{0}^{2 \pi} \int_{0}^{2 \pi} \int_{0}^{+\infty} s\left(z ; r, \phi, \phi_{0}\right) \ln \left(s\left(z ; r, \phi, \phi_{0}\right)\right) d r d \phi d \phi_{0} \\
& =\int_{0}^{2 \pi} \int_{0}^{2 \pi} \int_{0}^{+\infty} \lim _{z \rightarrow z_{0}} s\left(z ; r, \phi, \phi_{0}\right) \ln \left(s\left(z ; r, \phi, \phi_{0}\right)\right) d r d \phi d \phi_{0} \\
& =\int_{0}^{2 \pi} \int_{0}^{2 \pi} \int_{0}^{+\infty} s\left(z_{0} ; r, \phi, \phi_{0}\right) \ln \left(s\left(z_{0} ; r, \phi, \phi_{0}\right)\right) d r d \phi d \phi_{0} \\
& =u\left(z_{0}\right),
\end{aligned}
$$

where equation (68) holds since $s\left(z ; r, \phi, \phi_{0}\right) \ln \left(s\left(z ; r, \phi, \phi_{0}\right)\right)$ is continuous in $z$, considering the convention (41) when $s=$ 0 . We justify changing the order of the limit and the sum in (67) by finding, for large values of $r$, an upper bound on $\left|s\left(z ; r, \phi, \phi_{0}\right) \ln s\left(z ; r, \phi, \phi_{0}\right)\right|$ that is integrable in $\left(r, \phi, \phi_{0}\right)$ and independent of $z$. Let $\mathcal{B}\left(z_{0}, \zeta\right) \in \mathcal{O}$ be a neighborhood of 
$z_{0}$ and consider $z \in \mathcal{B}\left(z_{0}, \zeta\right)$. The bound in (65) implies that $\sum_{m=-\infty, m \neq 0}^{+\infty} C_{m}(r, z) e^{j m\left(\phi-\phi_{0}-\gamma z^{2} \mathcal{L}\right)}=o\left(\frac{1}{r^{2}}\right)$. Furthermore, using (4)

$$
\left|p_{R \mid R_{0}}(r \mid z)\right|=\frac{2 r}{\sigma^{2} \mathcal{L}}\left|e^{-\frac{r^{2}+z^{2}}{\sigma^{2} \mathcal{L}}}\right|\left|I_{0}\left(\frac{2 r z}{\sigma^{2} \mathcal{L}}\right)\right| \leq \frac{2 r}{\sigma^{2} \mathcal{L}} e^{-\frac{r^{2}}{\sigma^{2} \mathcal{L}}} e^{\frac{\left(\left|z_{0}\right|+\zeta\right)^{2}}{\sigma^{2} \mathcal{L}}} e^{\frac{2\left(\left|z_{0}\right|+\zeta\right) r}{\sigma^{2} \mathcal{L}}}=o\left(\frac{1}{r^{2}}\right),
$$

where we used Lemma 3 - 2 to write the inequality. Hence, equation 57 implies

$$
\left|s\left(z ; r, \phi, \phi_{0}\right)\right|=o\left(\frac{1}{r^{2}}\right)
$$

Note that, if $x \in \mathbb{C}$, then, since $\ln (x)=\ln |x|+j \arg (x),|\ln (x)| \equiv|\ln | x||$ as $x \rightarrow 0$. Thus $\mid s\left(z ; r, \phi, \phi_{0}\right) \ln \left(s\left(z ; r, \phi, \phi_{0}\right) \mid \equiv\right.$ ||$s\left(z ; r, \phi, \phi_{0}\right)|\ln | s\left(z ; r, \phi, \phi_{0}\right)||$ as $r \rightarrow+\infty$. We find an integrable upper bound on ||$s\left(z ; r, \phi, \phi_{0}\right)|\ln | s\left(z ; r, \phi, \phi_{0}\right)||$. When $r$ is large, $\left|s\left(z ; r, \phi, \phi_{0}\right)\right|<1$ and we apply the inequality (49) to obtain

$$
|| s\left(z ; r, \phi, \phi_{0}\right)|\ln | s\left(z ; r, \phi, \phi_{0}\right) \| \leq \frac{1}{1-\delta}\left|s\left(z ; r, \phi, \phi_{0}\right)\right|^{\delta},
$$

for any $\delta \in(0,1)$. Choosing $\delta \in(1 / 2,1)$ and using (70), we obtain an upper bound on (71) that is integrable in $\left(r, \phi, \phi_{0}\right)$ and independent of $z$.

For the second part of Morera's theorem, we consider the integral of $u(z)$ over the boundary $\partial \Delta$ of a compact triangle $\Delta \subset \mathcal{O}$. We have

$$
\begin{aligned}
\int_{\partial \Delta} u(z) d z & =\int_{0}^{2 \pi} \int_{0}^{2 \pi} \int_{0}^{+\infty} \int_{\partial \Delta} s\left(z ; r, \phi, \phi_{0}\right) \ln \left(s\left(z ; r, \phi, \phi_{0}\right)\right) d z d r d \phi d \phi_{0} \\
& =0 .
\end{aligned}
$$

Exchanging the integration order is justified from (70) and (71) by Fubini's theorem. It is shown in part 2) that $s\left(z ; r, \phi, \phi_{0}\right) \ln \left(s\left(z ; r, \phi, \phi_{0}\right)\right)$ is analytic in $z \in \mathcal{O}$. Hence, its integral over a closed contour in $\mathcal{O}$ is zero which justifies (72).

Equations (69) and (72) imply that $u(z)$ is analytic on $\mathcal{O}$ by Morera's theorem.

4) Analyticity of $\int_{0}^{+\infty} p_{R \mid R_{0}}(r \mid z) \ln \left(p_{R}\left(r ; F_{0}^{*}\right)\right) d r$ : The function

$$
p_{R \mid R_{0}}(r \mid z)=\frac{2 r}{\sigma^{2} \mathcal{L}} e^{-\frac{r^{2}+z^{2}}{\sigma^{2} \mathcal{L}}} I_{0}\left(\frac{2 r z}{\sigma^{2} \mathcal{L}}\right),
$$

is analytic in $z \in \mathbb{C}$. Consider

$$
w(z)=\int_{0}^{+\infty} p_{R \mid R_{0}}(r \mid z) \ln \left(p_{R}\left(r ; F_{0}^{*}\right)\right) d r .
$$

Applying the Morera's theorem and using the exponential decay of $p_{R \mid R_{0}}(r \mid z)$ in $r$ along with its analyticity in $z$, it can be shown that $w(z)$ is analytic on $\mathbb{C}$. The steps are similar to the proof of analyticity in part 3) above.

5) Analyticity of $\operatorname{LHS}_{A}(z)$ and $\operatorname{LHS}_{\rho}(z)$ : Let $\operatorname{LHS}_{A}(z)$ be an extension of $\operatorname{LHS}_{A}\left(r_{0}\right)$ (defined in (13)) to the complex plane:

$$
\operatorname{LHS}_{A}(z)=\nu(\mathcal{C}(z)-A)+C+\int_{0}^{+\infty} p(r \mid z) \ln p\left(r ; F_{0}^{*}\right) d r+\frac{1}{2 \pi} \int_{0}^{2 \pi} h\left(R, \Phi \mid z, \phi_{0}\right) d \phi_{0} .
$$

From the results of the parts 3) and 4) above, and the property C2 of $\mathcal{C}\left(r_{0}\right)$ stated in Section ஹ, we obtain that LHS ${ }_{A}(z)$ is analytic when $z \in \mathcal{O}_{\delta} \stackrel{\text { def }}{=} \mathcal{O} \cap \mathcal{S}_{\delta}$. In a similar manner, we have that $\operatorname{LHS}_{\rho}(z)$ (defined in (12)) is analytically extendable to $\mathcal{O}$, hence to $\mathcal{O}_{\delta}$.

\section{APPENDIX III}

\section{BOUNDS ON THE KKT CONDITIONS}

Lemma 10 (Upper Bound on $\mathbf{L H S}_{\rho}\left(r_{0}\right)$ ) Consider $\mathrm{LHS}_{\rho}\left(r_{0}\right)$ (defined in (12)) for large $r_{0}$. For any $0<\epsilon<1$, there exists a $K>0$ such that

$$
\operatorname{LHS}_{\rho}\left(r_{0}\right) \leq C+\ln \left(\frac{1}{K}\right)+\frac{r_{0}^{2}}{\sigma^{2} \mathcal{L}}-\ln \left(1-\xi\left(r_{0}\right)\right)+\left(\rho-(1-\epsilon) r_{0}\right) \sqrt{\frac{\pi}{\sigma^{2} \mathcal{L}}} L_{\frac{1}{2}}\left(-\frac{r_{0}^{2}}{\sigma^{2} \mathcal{L}}\right),
$$

where $\xi\left(r_{0}\right)$ is defined in Lemma 4 and $L_{\frac{1}{2}}(\cdot)$ is a Laguerre polynomial.

Proof: 
Let $F_{0}^{*} \stackrel{\text { def }}{=} F_{R_{0}^{*}}\left(r_{0}\right) \in \mathcal{P}$ be the optimal input cdf. Using the upper bound on $p\left(r, F_{0}^{*}\right)$ given in (39), we have

$$
\begin{aligned}
& \int_{0}^{+\infty} p_{R \mid R_{0}}\left(r \mid r_{0}\right) \ln p\left(r ; F_{0}^{*}\right) d r \\
& \leq \ln \left(\frac{2}{\sigma^{2} \mathcal{L}}\right)+\int_{0}^{\infty} \ln (r) p_{R \mid R_{0}}\left(r \mid r_{0}\right) d r-\frac{1}{\sigma^{2} \mathcal{L}} \int_{0}^{\infty} r^{2} p_{R \mid R_{0}}\left(r \mid r_{0}\right) d r+\frac{2 \rho}{\sigma^{2} \mathcal{L}} \int_{0}^{\infty} r p_{R \mid R_{0}}\left(r \mid r_{0}\right) d r \\
& =\ln \left(\frac{2}{\sigma^{2} \mathcal{L}}\right)+\int_{0}^{\infty} \ln (r) p_{R \mid R_{0}}\left(r \mid r_{0}\right) d r-\frac{1}{\sigma^{2} \mathcal{L}}\left(\sigma^{2} \mathcal{L}+r_{0}^{2}\right)+\rho \sqrt{\frac{\pi}{\sigma^{2} \mathcal{L}}} \mathrm{L}_{\frac{1}{2}}\left(-\frac{r_{0}^{2}}{\sigma^{2} \mathcal{L}}\right),
\end{aligned}
$$

where we used the fact that $p_{R \mid R_{0}}\left(r \mid r_{0}\right)$ is a Rician pdf with parameters $\left(r_{0}, \frac{\sigma^{2} \mathcal{L}}{2}\right)$. The first two moments of this pdf are

$$
\begin{aligned}
& \int_{0}^{\infty} r p_{R \mid R_{0}}\left(r \mid r_{0}\right) \mathrm{d} r=\frac{\sigma \sqrt{\pi \mathcal{L}}}{2} \mathrm{~L}_{\frac{1}{2}}\left(\frac{-r_{0}^{2}}{\sigma^{2} \mathcal{L}}\right), \\
& \int_{0}^{\infty} r^{2} p_{R \mid R_{0}}\left(r \mid r_{0}\right) \mathrm{d} r=\sigma^{2} \mathcal{L}+r_{0}^{2} .
\end{aligned}
$$

Furthermore, let $r_{0}>0$ be a large number and let $0<\epsilon<1$. Using the lower bound on the conditional pdf in Lemma 4 - 2 we have

$$
\begin{aligned}
& \frac{1}{2 \pi} \int_{0}^{2 \pi} h\left(R, \Phi \mid r_{0}, \phi_{0}\right) d \phi_{0} \\
& =-\frac{1}{2 \pi} \int_{0}^{2 \pi} \int_{0}^{+\infty} \int_{0}^{2 \pi} p\left(r, \phi \mid r_{0}, \phi_{0}\right) \ln p\left(r, \phi \mid r_{0}, \phi_{0}\right) d \phi d r d \phi_{0} \\
& \stackrel{(a)}{\leq} \ln (2 \pi)-\ln \left(1-\xi\left(r_{0}\right)\right)-\int_{0}^{+\infty} p_{R \mid R_{0}}\left(r \mid r_{0}\right) \ln \left(p_{R \mid R_{0}}\left(r \mid r_{0}\right)\right) d r \\
& =-\ln \left(\frac{1}{\pi \sigma^{2} \mathcal{L}}\right)-\int_{0}^{+\infty} \ln (r) p_{R \mid R_{0}}\left(r \mid r_{0}\right) d r+\frac{1}{\sigma^{2} \mathcal{L}}\left(r_{0}^{2}+\int_{0}^{+\infty} r^{2} p_{R \mid R_{0}}\left(r \mid r_{0}\right) d r\right) \\
& \leq \ln \left(\pi \sigma^{2} \mathcal{L}\right)-\int_{0}^{+\infty} \ln \left(I_{0}\left(\frac{2 r r_{0}}{\sigma^{2} \mathcal{L}}\right)\right) p_{R \mid R_{0}}\left(r \mid r_{0}\right) d r-\ln \left(1-\xi\left(r_{0}\right)\right) \\
& =\ln \left(\frac{\pi \sigma^{2} \mathcal{L}}{K}\right)-\int_{0}^{+\infty} \ln (r) p_{R \mid R_{0}}\left(r \mid r_{0}\right) d r-\ln \left(1-\xi\left(r_{0}\right)\right)+\frac{2 r_{0}^{2}+\sigma^{2} \mathcal{L}}{\sigma^{2} \mathcal{L}}-(1-\epsilon) \frac{2 r_{0}}{\sigma^{2} \mathcal{L}} \int_{0}^{+\infty} r p_{R \mid R_{0}}\left(r \mid r_{0}\right) d r \\
& =\ln \left(\frac{\pi \sigma^{2} \mathcal{L}}{K}\right)-\int_{0}^{+\infty} \ln (r) p_{R \mid R_{0}}\left(r \mid r_{0}\right) d r-\ln \left(1-\xi\left(r_{0}\right)\right)+\frac{1}{\sigma^{2} \mathcal{L}}\left(2 r_{0}^{2}+\sigma^{2} \mathcal{L}\right)-(1-\epsilon) r_{0} \sqrt{\frac{2 r r_{0}}{\sigma^{2} \mathcal{L}}} \mathrm{L}_{\frac{1}{2}}\left(-\frac{r_{0}}{\sigma_{0}}(r) R_{0}\left(r_{0}\right) d r\right.
\end{aligned}
$$

where step (a) is due to Lemma 4.2 and where we used Lemma 3 - 4 for some $K>0$, and equations (74) and (75) in order to write (77). Finally, combining (73) and (77), we have for sufficiently large $r_{0}$ :

$$
\begin{aligned}
& \operatorname{LHS}_{\rho}\left(r_{0}\right)= C-\ln (2 \pi)+\int_{0}^{+\infty} p\left(r \mid r_{0}\right) \ln p\left(r ; F_{0}^{*}\right) d r+\frac{1}{2 \pi} \int_{0}^{2 \pi} h\left(R, \Phi \mid r_{0}, \phi_{0}\right) d \phi_{0} \\
& \leq C-\ln (2 \pi)+\ln \left(\frac{2}{\sigma^{2} \mathcal{L}}\right)+\int_{0}^{\infty} \ln (r) p_{R \mid R_{0}}\left(r \mid r_{0}\right) d r-\frac{1}{\sigma^{2} \mathcal{L}}\left(\sigma^{2} \mathcal{L}+r_{0}^{2}\right) \\
&+\rho \sqrt{\frac{\pi}{\sigma^{2} \mathcal{L}}} \mathrm{L}_{\frac{1}{2}}\left(-\frac{r_{0}^{2}}{\sigma^{2} \mathcal{L}}\right)+\ln \left(\frac{\pi \sigma^{2} \mathcal{L}}{K}\right)-\int_{0}^{+\infty} \ln (r) p_{R \mid R_{0}}\left(r \mid r_{0}\right) d r-\ln \left(1-\xi\left(r_{0}\right)\right) \\
& \quad+\frac{1}{\sigma^{2} \mathcal{L}}\left(2 r_{0}^{2}+\sigma^{2} \mathcal{L}\right)-(1-\epsilon) r_{0} \sqrt{\frac{\pi}{\sigma^{2} \mathcal{L}}} \mathrm{L}_{\frac{1}{2}}\left(-\frac{r_{0}^{2}}{\sigma^{2} \mathcal{L}}\right) \\
&=C+\ln \left(\frac{1}{K}\right)+\frac{r_{0}^{2}}{\sigma^{2} \mathcal{L}}-\ln \left(1-\xi\left(r_{0}\right)\right)+\left(\rho-(1-\epsilon) r_{0}\right) \sqrt{\frac{\pi}{\sigma^{2} \mathcal{L}}} \mathrm{L}_{\frac{1}{2}}\left(-\frac{r_{0}^{2}}{\sigma^{2} \mathcal{L}}\right) .
\end{aligned}
$$

Lemma 11 (Lower Bound on $\operatorname{LHS}_{A}\left(r_{0}\right)$ ) The LHS of (13) satisfies:

$$
\operatorname{LHS}_{A}\left(r_{0}\right)>\nu\left(\mathcal{C}\left(r_{0}\right)-A\right)+C+\ln \left(\frac{k_{1}}{2 \pi k_{u}}\right)+\frac{1}{\sigma^{2} \mathcal{L}} r_{0}^{2}-r_{0} \sqrt{\frac{\pi}{\sigma^{2} \mathcal{L}}} L_{\frac{1}{2}}\left(-\frac{r_{0}^{2}}{\sigma^{2} \mathcal{L}}\right)
$$


where $k_{u}$ is defined in Lemma $4 k_{1}=\int_{0}^{+\infty} e^{-\frac{r_{0}^{2}}{\sigma^{2} \mathcal{L}}} d F_{R_{0}^{*}}\left(r_{0}\right)$, and $L_{\frac{1}{2}}(\cdot)$ is a Laguerre polynomial.

Proof:

Let $F_{0}^{*} \stackrel{\text { def }}{=} F_{R_{0}^{*}}\left(r_{0}\right) \in \mathcal{A}$ be the optimal input cdf. The first integral in $\operatorname{LHS}_{A}\left(r_{0}\right)$ is lower bounded by

$$
\int_{0}^{+\infty} p_{R \mid R_{0}}\left(r \mid r_{0}\right) \ln p\left(r ; F_{0}^{*}\right) d r \geq \int_{0}^{\infty} \ln (r) p_{R \mid R_{0}}\left(r \mid r_{0}\right) \mathrm{d} r+\ln \left(\frac{2 k_{1}}{\sigma^{2} \mathcal{L}}\right)-\frac{\sigma^{2} \mathcal{L}+r_{0}^{2}}{\sigma^{2} \mathcal{L}}
$$

where we used the lower bound on $p\left(r, F_{0}^{*}\right)$ in Lemma $5-3$ and (75).

The second integral in $\operatorname{LHS}_{A}\left(r_{0}\right)$ is lower bounded as

$$
\begin{aligned}
\int_{0}^{2 \pi} h\left(R, \Phi \mid r_{0}, \phi_{0}\right) d \phi_{0} & \stackrel{(b)}{>}-\int_{0}^{2 \pi+\infty} \int_{0}^{2 \pi} \int_{0}^{2 \pi} p\left(r, \phi \mid r_{0}, \phi_{0}\right) \ln \left(k_{u} p_{R \mid R_{0}}\left(r \mid r_{0}\right)\right) d \phi d r d \phi_{0} \\
& =-\int_{0}^{+\infty} \ln \left(k_{u} p_{R \mid R_{0}}\left(r \mid r_{0}\right)\right) \mathrm{d} r \int_{0}^{2 \pi} \int_{0}^{2 \pi} p\left(r, \phi \mid r_{0}, \phi_{0}\right) d \phi d \phi_{0} \\
& =-2 \pi \int_{0}^{+\infty} \ln \left(k_{u} p_{R \mid R_{0}}\left(r \mid r_{0}\right)\right) p_{R \mid R_{0}}\left(r \mid r_{0}\right) d r \\
& =2 \pi\left(h\left(R \mid r_{0}\right)-\ln \left(k_{u}\right)\right),
\end{aligned}
$$

where step $(b)$ is due to Lemma 4. 1 . From (4), $h\left(R \mid r_{0}\right)$ can be lower-bounded as:

$$
\begin{aligned}
h\left(R \mid r_{0}\right) & =-\int_{0}^{+\infty} p_{R \mid R_{0}}\left(r \mid r_{0}\right) \ln \left(p_{R \mid R_{0}}\left(r \mid r_{0}\right)\right) d r \\
& =-\ln \left(\frac{2}{\sigma^{2} \mathcal{L}}\right)+\frac{1}{\sigma^{2} \mathcal{L}}\left(r_{0}^{2}+\int_{0}^{+\infty} r^{2} p_{R \mid R_{0}}\left(r \mid r_{0}\right) d r\right)-\int_{0}^{+\infty} \ln \left(r I_{0}\left(\frac{2 r r_{0}}{\sigma^{2} \mathcal{L}}\right)\right) p_{R \mid R_{0}}\left(r \mid r_{0}\right) d r \\
& \geq \ln \left(\frac{\sigma^{2} \mathcal{L}}{2}\right)-\int_{0}^{+\infty} \ln (r) p_{R \mid R_{0}}\left(r \mid r_{0}\right) d r+\frac{1}{\sigma^{2} \mathcal{L}}\left(2 r_{0}^{2}+\sigma^{2} \mathcal{L}\right)-\frac{2 r_{0}}{\sigma^{2} \mathcal{L}} \int_{0}^{+\infty} r p_{R \mid R_{0}}\left(r \mid r_{0}\right) d r \\
& =\ln \left(\frac{e \sigma^{2} \mathcal{L}}{2}\right)-\int_{0}^{+\infty} \ln (r) p_{R \mid R_{0}}\left(r \mid r_{0}\right) d r+\frac{2}{\sigma^{2} \mathcal{L}} r_{0}^{2}-r_{0} \sqrt{\frac{\pi}{\sigma^{2} \mathcal{L}}} \mathrm{L}_{\frac{1}{2}}\left(-\frac{r_{0}^{2}}{\sigma^{2} \mathcal{L}}\right)
\end{aligned}
$$

where we used Lemma 3 - 2 in (80) and (74) in (81). Substituting (81) into (79)

$$
\frac{1}{2 \pi} \int_{0}^{2 \pi} h\left(R, \Phi \mid r_{0}, \phi_{0}\right) d \phi_{0}>\ln \left(\frac{e \sigma^{2} \mathcal{L}}{2 k_{u}}\right)+\frac{2}{\sigma^{2} \mathcal{L}} r_{0}^{2}-\int_{0}^{+\infty} \ln (r) p_{R \mid R_{0}}\left(r \mid r_{0}\right) d r-r_{0} \sqrt{\frac{\pi}{\sigma^{2} \mathcal{L}}} \mathrm{L}_{\frac{1}{2}}\left(-\frac{r_{0}^{2}}{\sigma^{2} \mathcal{L}}\right) .
$$

Finally, using lower bounds (78) and (82), we obtain

$$
\begin{aligned}
& \operatorname{LHS}_{A}\left(r_{0}\right)>\nu\left(\mathcal{C}\left(r_{0}\right)-A\right)+ C-\ln (2 \pi)+\ln \left(\frac{2 k_{1}}{\sigma^{2} \mathcal{L}}\right)+\ln \left(\frac{e \sigma^{2} \mathcal{L}}{2 k_{u}}\right) \\
&+\int_{0}^{+\infty} \ln (r) p_{R \mid R_{0}}\left(r \mid r_{0}\right) d r-\int_{0}^{+\infty} \ln (r) p_{R \mid R_{0}}\left(r \mid r_{0}\right) d r \\
& \quad-\frac{1}{\sigma^{2} \mathcal{L}}\left(\sigma^{2} \mathcal{L}+r_{0}^{2}\right)+\frac{2}{\sigma^{2} \mathcal{L}} r_{0}^{2}-r_{0} \sqrt{\frac{\pi}{\sigma^{2}}} \mathrm{~L}_{\frac{1}{2}}\left(-\frac{r_{0}^{2}}{\sigma^{2} \mathcal{L}}\right) \\
&=\nu\left(\mathcal{C}\left(r_{0}\right)-A\right)+C+\ln \left(\frac{k_{1}}{2 \pi k_{u}}\right)+\frac{1}{\sigma^{2} \mathcal{L}} r_{0}^{2}-r_{0} \sqrt{\frac{\pi}{\sigma^{2} \mathcal{L}}} \mathrm{L}_{\frac{1}{2}}\left(-\frac{r_{0}^{2}}{\sigma^{2} \mathcal{L}}\right) .
\end{aligned}
$$

\section{REFERENCES}

[1] G. Kramer, M. I. Yousefi, and F. Kschischang, "Upper bound on the capacity of a cascade of nonlinear and noisy channels," in IEEE Info. Theory Workshop, Jerusalem, Israel, Apr. 2015, pp. 1-4.

[2] M. I. Yousefi, G. Kramer, and F. R. Kschischang, "Upper bound on the capacity of the nonlinear Schrödinger channel," in Canadian Workshop on Inf. Theory, St. John's, Newfoundland, Canada, July 2015, pp. 1-5.

[3] M. I. Yousefi and F. R. Kschischang, "On the per-sample capacity of nondispersive optical fibers," IEEE Trans. Inf. Theory, vol. 57, no. 11, pp. 7522-7541, Nov. 2011.

[4] G. Agrawal, Nonlinear fiber optics. San Francisco, CA, USA: Academic, 2007.

[5] A. Mecozzi, "Limits to long-haul coherent transmission set by the Kerr nonlinearity and noise of the in-line amplifiers," IEEE J. Lightw. Technol., vol. 12, no. 11, pp. 1993-2000, Nov. 1994. 
[6] K. S. Turitsyn, S. A. Derevyanko, I. V. Yurkevich, and S. K. Turitsyn, "Information capacity of optical fiber channels with zero average dispersion," Phys. Rev. Lett., vol. 91, no. 20, p. 203901, Nov. 2003.

[7] J. G. Smith, "The information capacity of amplitude- and variance-constrained scalar Gaussian channels," Inf. Contr., vol. 18, no. 3, pp. 203-219, Apr. 1971.

[8] W. Hirt and J. Massey, "Capacity of the discrete-time Gaussian channel with intersymbol interference," IEEE Trans. Inf. Theory, vol. 34, no. 3, pp. 380-388, May 1988.

[9] S. Shamai and I. Bar-David, "The capacity of average and peak-power-limited quadrature Gaussian channels," IEEE Trans. Inf. Theory, vol. 41, no. 4, pp. 1060-1071, July 1995.

[10] A. Das, "Capacity-achieving distributions for non-Gaussian additive noise channels," in IEEE Int. Symp. Info Theory, Sorrento, Italy, June 2000 , p. 432.

[11] I. Abou-Faycal, M. D. Trott, and S. Shamai, "The capacity of discrete-time memoryless Rayleigh-fading channels," IEEE Trans. Inf. Theory, vol. 47, no. 4, pp. 1290-1301, May 2001.

[12] A. Tchamkerten, "On the discreteness of capacity-achieving distributions," IEEE Trans. Inf. Theory, vol. 50, no. 11, pp. 2773-2778, Nov. 2004

[13] T. H. Chan, S. Hranilovic, and F. Kschischang, "Capacity-achieving probability measure for conditionally Gaussian channels with bounded inputs," IEEE Trans. Inf. Theory, vol. 51, no. 6, pp. 2073-2088, June 2005.

[14] M. G. Gursoy, H. V. Poor, and S. Verdu, "The noncoherent Rician fading channel-Part I: structure of the capacity-achieving input," IEEE Trans. Wireless Commun., vol. 4, no. 5, pp. 2193-2206, Sept. 2005.

[15] J. Fahs and I. Abou-Faycal, "Using Hermite bases in studying capacity-achieving distributions over AWGN channels," IEEE Trans. Inf. Theory, vol. 58, no. 8, pp. 5302-5322, Aug. 2012.

[16] J. Fahs, N. Ajeeb, and I. Abou-Faycal, "The capacity of average power constrained additive non-Gaussian noise channels," in IEEE Int. Conf. Telecommun., Beirut, Lebanon, Apr. 2012.

[17] J. Fahs and I. Abou-Faycal, "On properties of the support of capacity-achieving distributions for additive noise channel models with input cost constraints," IEEE Trans. Inf. Theory, vol. 58, no. 2, pp. 1178 - 1198, Feb. 2018.

[18] G. Kramer, "Autocorrelation function for dispersion-free fiber channels with distributed amplification," IEEE Trans. Inf. Theory, pp. 5131-5155, July 2018.

[19] J. Fahs and I. Abou-Faycal, "On the finiteness of the capacity of continuous channels," IEEE Trans. Commun., vol. 64, no. 1, pp. 166-173, Jan. 2016.

[20] W. Rudin et al., Principles of mathematical analysis. McGraw-hill New York, 1964, vol. 3.

[21] H. Silverman, Complex Variables. Boston, Massachusetts, USA: Houghton Mifflin Company, 1975.

[22] M. Abramowitz and I. A. Stegun, Handbook of Mathematical Functions with Formulas, Graphs, and Mathematical Tables, 10th ed., ser. Appl. Math. Washington, D.C., USA: National Bureau of Standards, 1972.

[23] M. I. Yousefi, "The asymptotic capacity of the optical fiber," Arxiv preprint, arxiv:1610.06458, pp. 1-12, Nov. 2016. 